\title{
Reversible Electronic Logic Using Switches
}

by

\author{
Ralph C. Merkle \\ Xerox PARC \\ 3333 Coyote Hill Road \\ Palo Alto, CA 94304 \\ merkle@xerox.com \\ ftp://parcftp.xerox.com/pub/merkle/merklesHomePage.html
}

Copyright 1990 by Xerox Corporation.

All Rights Reserved.

This article was published in Nanotechnology, Volume 4, 1993, pages 21 through 40.

This version differs in minor respects from the published version.

Nanotechnology, published by IOP (the Institute of Physics), is available to individual subscribers in the US for a subscription rate of $\$ 111$ U.S. dollars; (or 45 British pounds in the EC, 54 pounds outside the EC, as of 1994). Subscription and other information is available from ftp://parcftp.xerox.com/pub/nano/IOP.html, or fax IOP at 516-349-7669 (Europe: 0272 294318; Tokyo, Japan 278 1937).

\begin{abstract}
The continuing revolution in computer hardware is packing ever more logic gates in ever smaller volumes. Unfortunately this produces ever more heat, which will limit the feasible packing density and performance unless the energy dissipated by each logic operation can also be dramatically reduced. To reduce the energy dissipation of a logic operation below $\ln (2) k T$ (near thermal noise) requires the use of reversible logic for fundamental thermodynamic reasons. Extrapolation of current trends suggest this limit will become significant within one to two decades. Many real devices can be viewed as electrically controlled switches, so a method of using an abstract switch in a reversible manner is useful. Two methods of using switches to implement reversible computations are discussed in this paper. The first method has an energy dissipation which is proportional to the square of the error in the voltage, while the second method has an energy dissipation which can in principle be reduced indefinitely by slowing the speed of computation. The first method is basically an extension to "pass logic" which has been previously used with both nMOS (hot clock nMOS) and CMOS transmission gates to achieve low energy dissipation. The second method is a novel thermodynamically reversible logic system based on CCD-like operations which switches charge packets in a reversible fashion to achieve low energy dissipation.
\end{abstract}




\section{Introduction}

There is now a fairly extensive literature on reversible computation[11] which shows that the energy dissipation per device operation cannot be reduced below $\ln (2) k T$ (where $k$ is Boltzman's constant and $T$ is the temperature; $k T$ is roughly the thermal energy of a single atom) if the device is not reversible.

For the last 50 years the energy dissipation per gate operation has been declining with remarkable regularity[6]. Extrapolation of this trend shows the energy dissipation per device operation reaching $k T$ by the year 2015. (This assumes that $T$ is 300 Kelvin - more on this later). To gain some perspective on this consider that an "AND" gate which has a power supply of one volt and which allows 100 electrons to go from that one volt supply to ground during the course of a switching operation will dissipate 100 electron volts. Although 100 electron volts is about 4,000 times $k T$ (and well above the theoretical limit), it will be difficult for simple improvements of irreversible approaches to reach even this level of energy dissipation. Extrapolating present trends, we should reach 4,000 $k T$ before the year 2000, e.g., within ten years. While reversible logic is an absolute necessity if we are to reduce energy dissipation per device operation below $k T$, it is a useful heuristic for designing systems that have low energy dissipation even when the actual energy dissipation is well above $k T$.

Even if we do develop irreversible devices that approach $\ln (2) k T$, a computer operating at room temperature at a frequency of one gigahertz with $10^{18}$ logic gates packed into a volume not much larger than a cubic centimeter would dissipate over three megawatts. The drive for ever greater computational power with ever more densely packed logic elements will eventually require that a single logic operation dissipate orders of magnitude energy less than $k T$. If we are to realize the full potential of nanoelectronics and molecular logic devices, at some point in the future we will be forced to use thermodynamically reversible logic elements for a substantial fraction of logic operations.

We can state quite confidently that one of three things will occur: (a) the historic rate of decreasing energy dissipation per device operation will slow or halt in the next one or two decades or (b) we will operate computers at lower temperatures or (c) we will develop new methods of computing in a reversible way that can beat the $k T$ barrier.

The first option is unattractive. First, cooling is a major problem. The heroic cooling methods used in the Cray 3 supercomputer to remove the heat generated by the computer's operation suggest that failure to reduce energy dissipation per gate operation would be a major limiting factor in future computer performance. While both air-cooling a single chip that dissipates 150 watts[62] and water cooling of a chip dissipating 790 watts $/ \mathrm{cm}^{2}$ have been demonstrated[64], and more effective cooling methods should be feasible in the future[18], we will eventually reach a limit to heat removal. Second, in many applications power is limited. Portable computers, remote sensors and other isolated systems have only a limited amount of power available. Third, although the raw cost of electrical power is not yet a major limitation it would become so in the future if reductions in energy dissipation did not keep pace with advances in other areas. The Wall Street Journal[63] said "Computer systems currently account for $5 \%$ of commercial electricity use in the U.S., with the potential to grow to $10 \%$ by the year $2000 . "$ 
Operating computers at a lower temperature will not reduce overall energy dissipation. If we operated future devices at 3 Kelvins we would reduce $k T$ by a factor of 100 and so could reduce energy dissipation per logic operation by a similar factor - but for fundamental thermodynamic reasons the coefficient of performance of the refrigerator for the system can be at best $3 \mathrm{~K} /(300 \mathrm{~K}-$ $3 \mathrm{~K}) \approx 0.01[5]$. Thus, the lower energy required per gate operation will be almost exactly balanced by the increased energy needed by the refrigerator. In many applications refrigeration is not an attractive option. Lap top and portable computers[59], embedded systems, various "smart" appliances and other applications make the use of refrigeration undesirable.

Factors other than net energy savings can make low temperature operation worthwhile. For example, some potentially attractive devices don't operate at higher temperatures. In large computers operating in stable environments (the traditional computer center, for example) refrigeration might be attractive, particularly if it permits the use of devices that provide much better performance but which require low temperature for their operation (Josephson junction devices might be an example). Refrigeration per se, however, does not seem too attractive.

Finally, and most attractively, we could use reversible computation to reduce energy dissipation. This would, in principle, allow energy dissipations indefinitely below $k T$ per logic operation. While some barrier should eventually be encountered, the use of reversible logic should allow us to continue current trends in energy dissipation per logic operation for the longest possible time. It is interesting to note that certain uses of pass logic, transmission gates, and hot clock nMOS do in fact perform some logic operations in a thermodynamically reversible fashion. Research in the design and utilization of reversible logic to futher lower energy dissipation will simply recognize and make explicit a trend that has already begun. Further research in this area is the most appropriate response to the rather limited range of possibilities that face us.

\section{Reversible Architectures}

One concern about this approach is the need to use reversible computer architectures. Such architectures are entirely feasible[6, 7, 8, 11, 15, 19]. A wide variety of computations can be done in a logically reversible manner. Bennett[15] concluded: “...for any $\varepsilon>0$, ordinary multitape Turing machines using time $T$ and space $S$ can be simulated by reversible ones using time $\mathrm{O}\left(T^{1+\varepsilon}\right)$ and space $\mathrm{O}(S \log T)$ or in linear time and space $\mathrm{O}\left(S T^{\mathfrak{E}}\right)$." This general result shows that even arbitrary irreversible computations can be mapped into reversible computations. Specific irreversible computations can often be mapped into reversible computations with little or no loss of efficiency. Even if we do not adopt new reversible computer architectures, simple applications of reversible computation could be made within the framework of existing architectures. A typical computer executes a sequence of instructions, and each instruction will typically change the contents of a single register or memory location. Although loading the result of the instruction into a register will normally be irreversible (it erases the previous contents of the register) it is still the case that all other operations performed by the computer during instruction execution could in principle be made reversible. Thus, although we would have to dissipate roughly $k T$ energy for each bit in the output register for each instruction execution, we need not use dissipative logic throughout the computer (as is currently done).

We can go one step further without making significant changes in computer architecture: the simple 


\section{INPUT}

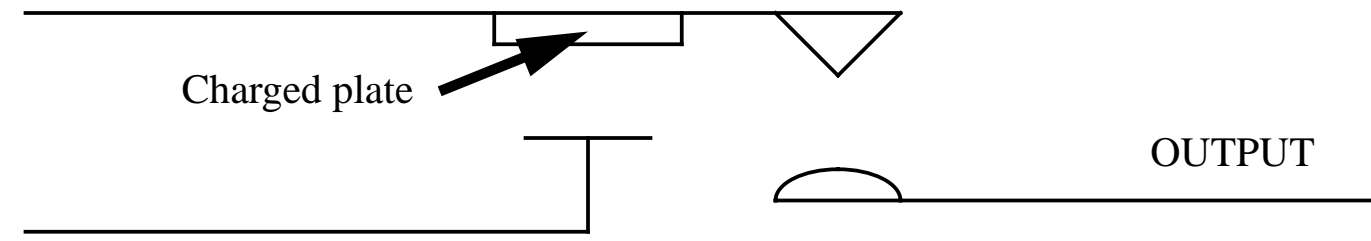

CONTROL Figure 1. An Electrostatic Voltage

Controlled Switch

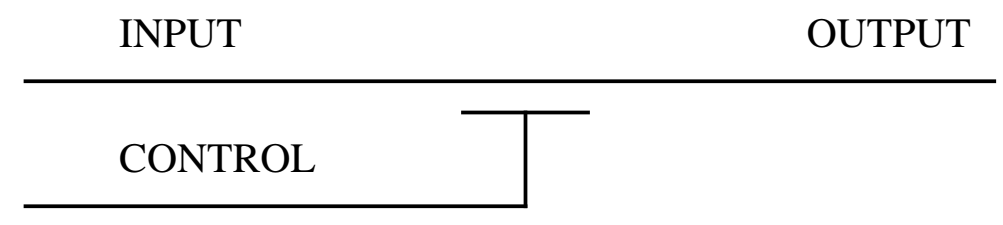

Figure 2. Symbol for a Voltage Controlled Switch

register-register add instruction $\mathrm{R} 1=\mathrm{R} 1+\mathrm{R} 2$ is logically reversible. With proper hardware, this particular instruction could in principle be made to dissipate as little energy as we desired. While non-reversible instructions (e.g., R1 =0) would still dissipate greater energy, the overall energy dissipation of the computer could be reduced significantly if a reversible approach were developed and used to implement the reversible instructions. Of course, once the energy-wasting instructions were identified, compilers would learn to avoid using them. Today, compilers can optimize for speed of computation and for the amount of memory used. In the future, compilers could also optimize a program to minimize the energy dissipated during computation, e.g., to minimize the number of bits that are erased. This would provide an entirely evolutionary path from the current irreversible designs to computer architectures that were as reversible as was practically feasible. While it is perhaps not obvious how far this trend can go it is clear that a very large percentage of computer operations can be made reversible - perhaps a remarkably large percentage.

\section{Reversible Devices}

Several reversible devices have been proposed over the years. Von Neumann and independently Goto proposed the parametron[37] which encodes information in the phase of an oscillation. Fredkin and Toffoli[23] proposed an electronic reversible logic family which used switches, capacitors and inductors. By turning the switches on and off at just the right times, charge could be transferred between the capacitors through the inductors with minimal energy loss. By increasing the size of the inductors it would be possible to create a family of ever slower but ever more energy efficient 
circuits. As the LC time constant is increased, the energy dissipation can be decreased to an arbitrary extent. A proposal by Likharev based on Josephson junctions $[9,10]$ “...is particularly significant, because it is a genuine example ... of a system that has frictional forces proportional to velocity" according to Landauer[6]. The parametric quantron is based on Josephson junctions and operates at low temperatures. It should dissipate less than $k T$ when operating at 4 kelvins and with a switching time of 1 nanosecond[9]. Modern "high temperature" superconductors might allow operation at relatively high temperatures, including that of liquid nitrogen[45]. Even these temperatures would still be a significant disadvantage. Molecular-scale mechanical reversible "rod logic" has been proposed by Drexler which should dissipate less than $k T$ when operating at room temperature at a speed of 50 to 100 nanoseconds $[2,18]$. Drexler's proposal is also significantly smaller than the parametric quantron. While the ability to fabricate Drexler's proposal is some decades away, it provides a good argument that molecular-scale reversible devices should be feasible.

Although the parametric quantron can be used in a reversible fashion to achieve low energy dissipation it uses magnetic fields to couple logic devices, requires low temperatures to operate and will likely prove resistant to scaling to the molecular size range. By contrast, Drexler's mechanical proposal might well prove smaller than even the smallest of future electronic devices. Mechanical devices depend fundamentally on the position of the nuclei of the atoms of which they are made. The position of the nucleus of an atom can be much more precisely known than the position of an electron: quantum uncertainty in the position of the electron is much greater because the mass of the electron is much smaller. If device function depends on the position of an electron then device size will be larger than if device function depends on the position of the nucleus. Such molecular mechanical proposals will, however, almost certainly be slower because of the greater mass of the nuclei of atoms as compared with the mass of electrons. In this paper we discuss simple methods of using relatively conventional electronic switching devices in a reversible fashion which can be scaled to a very small size. The methods described here allow a simple voltage-controlled switch to be used in a reversible manner, both to implement combinational logic and also to iterate an arbitrary reversible logic function. Other methods are feasible. For example, helical logic -- an elegant form of reversible electronic logic -- has recently been proposed by Merkle and Drexler[67].

Voltage controlled switches of various types have long been used in electronic devices[56, 66]. Not all voltage controlled switches are suitable for use in low energy designs because of their intrinsic energy dissipation. Traditional relays actuated by electromagnets dissipate comparatively large amounts of energy. Even if a switch were suited for low energy operation it would still dissipate energy if it were turned on when there was a voltage across it. The abrupt rush of current that normally occurs when a switch is turned on would insure this. Most previous logic designs based on switches operated in this manner (though the proposal by Fredkin and Toffoli[23] avoids this problem, and the proposal by Seitz et. al. [29] eliminates dissipative switch closures for combinational logic, although not for sequential logic). The switch is turned on for the purpose of draining charge when the charge is no longer wanted. That is, a logic " 1 " might be encoded by a charge on a capacitor. Turning on a switch to drain away that charge would dissipate energy even if the switch itself were perfectly non-dissipative.

A related but distinct line of research also emphasizes the importance of energy dissipation, but assumes that the losses involved in dissipatively switching a wire from one logic state to another are fixed and cannot be altered, e.g., that slowing the switching speed will not reduce energy dis- 
sipation. Even assuming that this is true, clever design of the circuit can greatly reduce the number of times it is necessary to take such a dissipative action[52,65]. Further research which combines both this approach and the approaches described here would seem fruitful.

Even relatively recently some authors have argued that switching devices must fundamentally dissipate more than $k T$ regardless of how slowly they operate[57]. It is worth noting, therefore, that charging and discharging a condenser need not dissipate the energy involved. For example, a simple LC oscillator will repeatedly charge and discharge a condenser. Although the energy stored on the condenser is $1 / 2 \mathrm{CV}^{2}$, this energy need not be dissipated during a charge/discharge cycle. If the $\mathrm{Q}$ of the circuit is high the energy dissipation per cycle will be small. In addition, the energy dissipation of such an oscillator depends on frequency. By using larger condensers and inductors the frequency of operation can be slowed and the fraction of energy dissipated per cycle reduced (see Drexler[18] for a discussion of scaling laws). If all linear dimensions of a device are scaled in size in proportion to some characteristic length $\mathrm{L}$ then capacitance and inductance are proportional to $\mathrm{L}$ while frequency and resistance are proportional to $1 / \mathrm{L}$. The $\mathrm{Q}$ of the circuit is proportional to L so the percentage of the total energy in the oscillator that is dissipated per cycle can be reduced simply by increasing all linear dimensions equally. Different scaling methods can produce better results.

If we assume that a fixed capacitive load is charged through a fixed resistance by a voltage that is ramped up over a period of time $\mathrm{T}$, then the energy dissipated by the resistance is approximately $1 / 2 \mathrm{CV}^{2} \times 2 \mathrm{RC} / \mathrm{T}$ (for $\mathrm{RC} \ll \mathrm{T}$ ). This dissipation can be reduced as much as we might want by increasing $\mathrm{T}$ (and leaving the circuit components unchanged). Koller and Athas[61] have called this adiabatic charging[61]. We will call it "thermodynamically reversible" or simply "reversible," relying on context to make it clear that we are referring to an asymptotically non-dissipative process. Note that the term "reversible" can also be used to mean "logically reversible" with no implication that it is asymptotically non-dissipative. A logically reversible process might be highly dissipative, so it is sometimes necessary to distinguish between "logically reversible" and "thermodynamically reversible." The term "reversible" in this paper will usually mean "thermodynamically reversible and asymptotically non-dissipative." The reversible charging of a condenser is illustrated in figure 3.

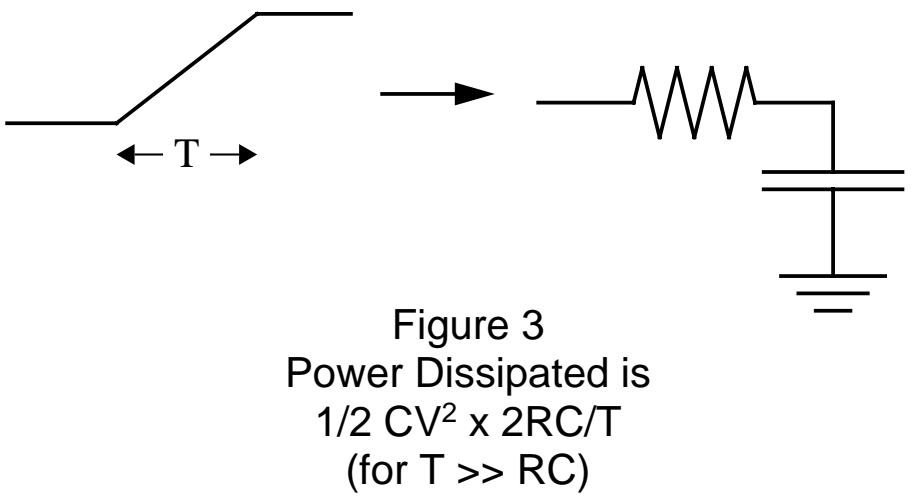

The situation is quite different for a step function in which the voltage is ramped up instantly $(T=0)$. This process dissipates $1 / 2 \mathrm{CV}^{2}$. Charge falls through the fixed potential drop in a dissipative fashion much as water goes over Niagra falls. This is illustrated in figure 4. Although this is the meth- 


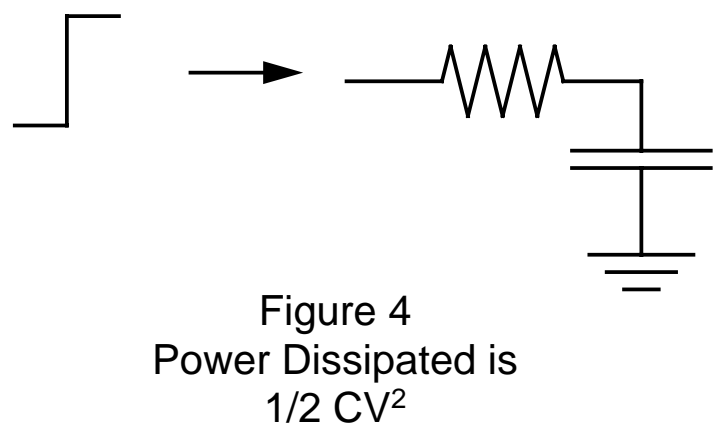

od in common use today to charge and discharge the various capacitive loads in computer circuits, it is not the method that we will be considering.

Comparing figures 3 and 4 shows that the advantage of reversible charging is in the factor of $2 \mathrm{RC} / \mathrm{T}$. Normally the switching time $\mathrm{T}$ must be longer than the RC time constant of the circuit simply to insure that the output has time to settle to its proper value. By using reversible charging, further increases in the switching time can be used to decrease the energy dissipation per logic operation. This, of course, results in a slower speed of operation leading to a trade-off between speed and energy dissipation.

How to arrange matters so that current flows smoothly and reversibly is the principle topic of the rest of the paper. This is challenging because the basic switching devices are (1) abrupt and discontinuous and (2) pass through a dissipative intermediate state (which is neither fully on nor fully off) while switching.

\section{The First Method}

The first method uses a conventional voltage controlled switch but in a somewhat unusual manner. In essence we never turn a switch on or off when there is either a voltage across it or current going through it. This mode of operation is reversible.

This approach has previously been used to compute combinational functions e.g., appropriately implemented pass logic [55, 57] or CMOS transmission gates [53]; see Seitz et. al.[29] for a description of hot clock nMOS. We extend this approach to implement a sequential circuit which can iterate the computation of a reversible logic function. Several authors have pursued this approach recently, including van de Snepscheut[60], Koller and Athas[61], and Hall[21], and have reached similar conclusions. It is also related to "dry switching," a method for using relays which avoids turning relays on or off when there is a voltage across them[68]. The primary purpose of dry switching is to avoid deterioration of the relay contacts, rather than minimize switching energy.

Conceptually, a voltage controlled switch can be thought of as a relay which is controlled electrostatically, as illustrated in Figure 1. We adopt the symbol shown in Figure 2 for a voltage controlled switch. We also adopt the following convention for turning a switch on and off: when the control input to the switch is at logic "0" (low) the switch is turned on and current can flow from the input to the output or from the output to the input. When the control input is "1" (high) the switch is turned off and current cannot flow. 
The reader should note that this convention is not the usual one. We adopt it instead of its opposite - where a " 1 " turns the switch on and a " 0 " turns it off-- because it is more intuitive when applied to the second proposal discussed later in this paper, the CCD-based reversible logic element.

The proposed mode of operation is fundamentally clocked and multi-staged. The outputs of stage $i$ drive the inputs of stage $i+1$. For the moment, we assume the output of stage 0 is simply given. That is, a set of input lines are assumed to have fixed voltages upon them representing the input data. The value of the succesive logic levels can then be computed in turn. The logic values (voltages) produced by stage 1 are computed from the logic values given by stage 0 ; The logic values produced by stage 2 are computed from the logic values produced by stage 1; etc. etc.

Simple pass logic can be employed to implement combinational logic. A "NOR" operation in this logic is simply two switches in series. A clock signal (initially at logic 0 ) is connected to the input of the first switch. If the control inputs of both switches have settled to logic 0 , then both switches will conduct. The clock input connected to the first switch is slowly raised (from logic 0 to logic 1). Both switches will conduct and the output will gradually shift to a logic 1 . If either input is 1 , one of the switches will not conduct and the output will remain at 0 . Because the clock signal can be raised and lowered slowly and reversibly, energy dissipation can in principle be made as small as desired for this operation simply by slowing down the speed with which we clock the circuit. A simple "NOR" gate is illustrated in figure 5. A "NAND" gate is illustrated in figure 6.

\section{Making an N-level Circuit}

We assume that we have an $\mathrm{N}$ level combinational circuit, and that $\mathrm{N}$ "clock signals" are provided. Initially, clocks 1 through $\mathrm{N}$ are low (logic 0 ), and the outputs of stage 0 are at some preset values. The outputs from stage 1 are computed as simple logical functions from the outputs of stage 0 . The outputs from stage $i$ are used as the inputs to stage $i+1$. A "NOR" and "NAND" gate from stage 1 are shown in Figures 5 and 6. The NAND gate is logically complete so any arbitrary boolean function can be implemented by using an appropriately connected collection of them.

The clock signals are now raised in turn. The output of "stage 0" is assumed to be valid prior to the start of operations, and clock 0 is unspecified (for now). Clock 1 is raised, and the outputs from stage 1 become valid. Clock 2 is raised, and the outputs from stage 2 become valid. This sequence continues, until clock $\mathrm{N}$ is raised, and the outputs from stage $\mathrm{N}$ become valid.

A single stage in the $\mathrm{N}$ level circuit could in principle implement an arbitrary boolean switching function by an appropriate arrangement of switches. This might require an exponential number of switches and so multiple stages appear to be useful. Also, although for simplicity we have assumed that the outputs of stage $i$ are used directly only in stage $i+1$, they could equally be used in stages $\mathrm{i}+2, \mathrm{i}+3$, etc. We will not consider these additional complexities here because they are secondary to the fundamental issues.

\section{Latches}

What has been described so far will allow the construction of an $\mathrm{N}$ level reversible combinational logic circuit. To be useful, we must also specify a latch which can store the output of the circuit. This can be done by holding charge on a capacitor. The outputs of stage $\mathrm{N}$ are connected through 
STAGE 1

CLOCK 1

OUTPUT

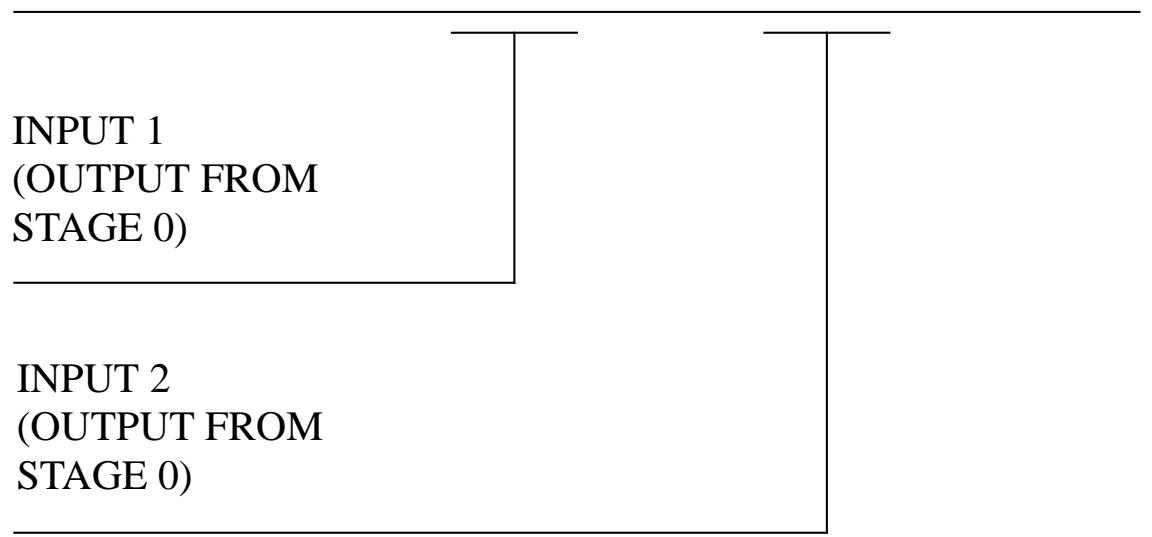

Figure 5. NOR gate

STAGE 1

CLOCK 1

OUTPUT

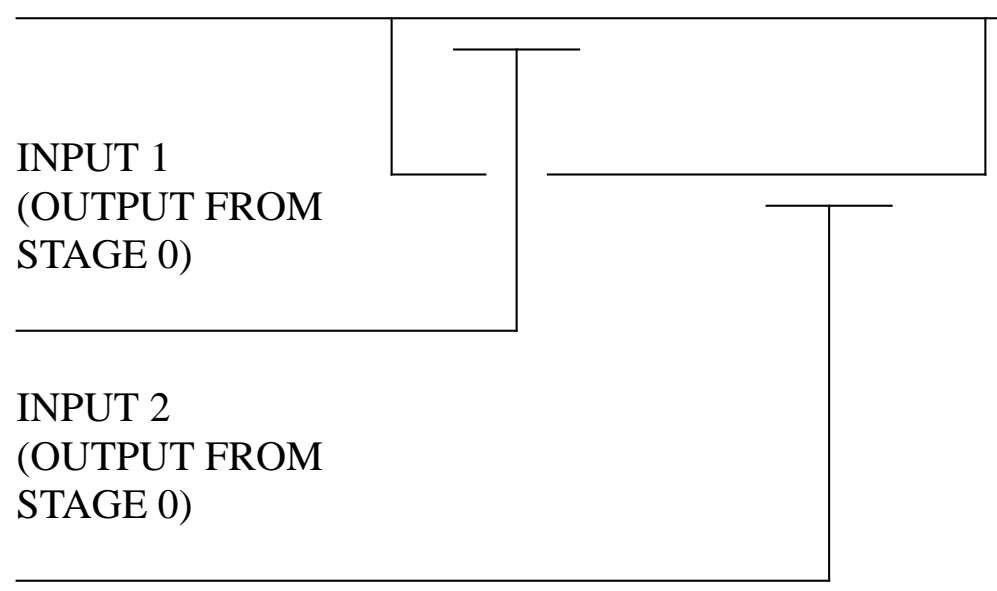

Figure 6. NAND gate

switches to a latch stage, stage $\mathrm{N}+1$. The latch requires its own clock. Such a latch is shown in figure 7 .

Initially, the latch clock is 0 and allows charge to move freely into the capacitor. When the output from stage $\mathrm{N}$ is valid, the latch clock goes high (changes to logic 1) and prevents charge from escaping. Clock $\mathrm{N}$ can now go low (to logic 0 ) which will make the output from stage $\mathrm{N}$ also go low, but the output of the latch will not be effected by changes in the output from stage $\mathrm{N}$ because it is cut off. 


\section{LATCH CLOCK}

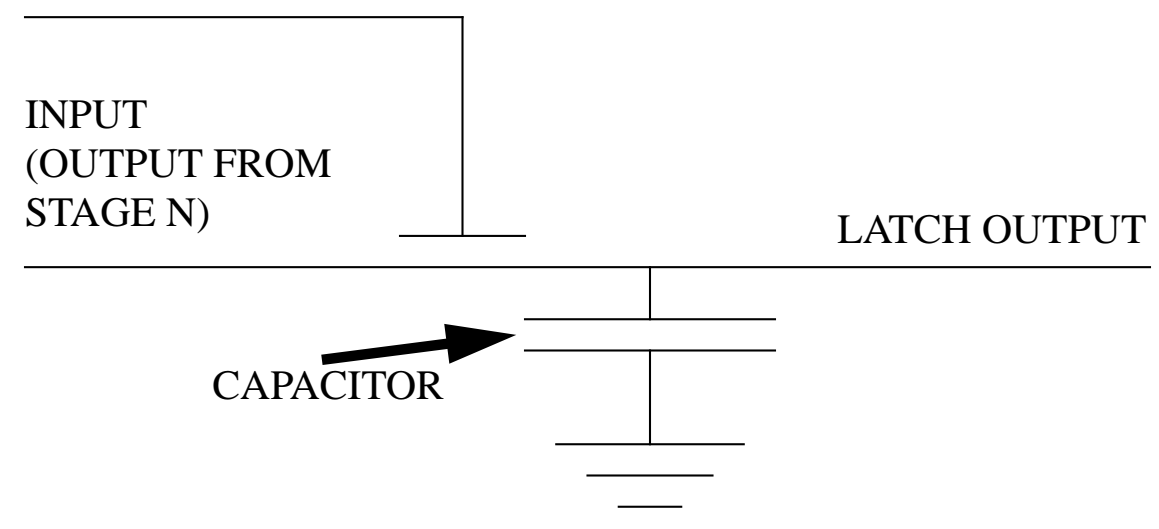

Figure 7. A latch

Of course, having once "locked up" the outputs in the latches, we cannot release the information in the latches. If we should again set the clock latch low, allowing charge to flow off the capacitor, then we would be turning on a switch when there was a voltage across it. This is forbidden, and so the problem of removing information from a latch is non-trivial.

\section{Unwriting}

Rather than simply "erasing" the information on the latch by letting the latch clock go low, we need to "unwrite" the information. Unwriting differs fundamentally from erasing. When we erase, we do not know the state of the latch, we simply turn on the switch holding the charge on the latch and let the charge flow away. Whether the latch held a " 0 " or a " 1 " is immaterial. In the process, we must necessarily dissipate energy because erasing information is irreversible.

By contrast, when we "unwrite" a latch we actually have an input signal that tells us the state of the latch. We can set the latch clock low and connect the input signal and the value stored on the latch. Because (by definition) the input signal is in the same logical state as the latch, there is no voltage across the switch when we turn it on. We can now let the input signal go to 0 slowly, thus draining charge from the latch. We can indefinitely reduce the energy dissipated by this process by slowing the speed with which we drain charge from the latch.

\section{Summary of Combinational Logic}

In our earlier description, we left the source of valid input a mystery. The outputs of stage 0 held valid data, and how this happy state of affairs came about was unspecified. Now we can rectify this omission: stage 0 is a latch. This implies that clock 0 must be kept high to prevent the charges in stage 0 from leaking away. Further, stage $\mathrm{N}+1$ is a latch, and holds the output. We still leave the mechanism that initially put proper charge levels into the capacitors of stage 0 a mystery, but it is now clear that stage 0 will continue to hold a valid signal until clock 0 is lowered and the charge is allowed to escape. The latches of stage 0 will also be called the input latches or simply the input, 
while the latches of stage $\mathrm{N}+1$ will also be called the output latches or simply the output.

We can now specify our initial state as one in which appropriate charges are held on the input, clock 0 is high (to prevent charge from the input latches from leaking away), while clocks 1 through $\mathrm{N}$ are held low; as is clock signal $\mathrm{N}+1$ (that is, there are no charges held on the output latches and the switch connecting the output latches to the final logic stage is turned on). We raise clock signals 1 through $\mathrm{N}$ in turn, computing the values of each logic level in turn. Finally, we raise clock signal $\mathrm{N}+1$, thus trapping the output signals in the output latches. We are then free to lower clock signal $\mathrm{N}$, then $\mathrm{N}-1$, then $\mathrm{N}-2$, etc. until we have finally worked our way back to the first logic level. When the computation is done, all clock signals from 1 through $\mathrm{N}$ are held low, while clock signals 0 and $\mathrm{N}+1$ remain high. Charge is trapped in stage 0 corresponding to the input, and charge is trapped in stage $\mathrm{N}+1$ corresponding to the output. The outputs of stage 0 and $\mathrm{N}+1$ are valid. All other outputs are 0 .

\section{Iteration of a Reversible Logic Function}

At a higher level of abstraction, we can say that the outputs of stage $\mathrm{N}+1$ are equal to some combinational function $\mathrm{F}$ applied to the outputs of stage 0 , or output $=\mathrm{F}$ (input). Of course, we're now stuck. We cannot unwrite the input latches because we don't know what information is stored in them.

We can solve this dilemma by requiring that $\mathrm{F}$ be reversible. That is, we demand that $\mathrm{F}^{-1}$ ( $\mathrm{F}$ (input)) $=$ input for all possible values of the input on stage 0 . This allows us to compute the values stored in stage 0 from the values stored in stage $\mathrm{N}+1$. We need merely compute $\mathrm{F}^{-1}$ (output). That is, we add additional logic stages $\mathrm{N}+2, \mathrm{~N}+3, \ldots, \mathrm{N}+\mathrm{M}$ which compute the inverse of $\mathrm{F}$. The output of stage $\mathrm{N}+\mathrm{M}$ will then be the same as the input. Because we now have data values which are identical to the contents of stage 0 , we can unwrite stage 0 in a fully reversible fashion. That is, we can lower clock 0 and allow the charge on the capacitors in stage 0 to be connected with the output signals from stage $\mathrm{N}+\mathrm{M}$. Because they are identical the energy loss from this operation can be made arbitrarily small. Then, clock signal $\mathrm{N}+\mathrm{M}$ can be lowered, clock signal $\mathrm{N}+\mathrm{M}-1$ can be lowered, etc. until we finally lower clock signal $\mathrm{N}+2$. Clock signal $\mathrm{N}+1$ (the latch control clock signal for the output) is high (to hold the output steady in the output latches) and remains high.

To review the cycle of operations from the initial state: we start with a valid input in stage 0 , with clock signal 0 held high (to hold the charge in the latches of stage 0 ) and with all other clock signals held low. From this intial state, we start the computation by raising clock signals 1 through $\mathrm{N}$ in turn, computing $\mathrm{F}$ (input). Clock signal $\mathrm{N}+1$ is then raised to hold the output values in the output latches of stage $\mathrm{N}+1$, and then clock signals $\mathrm{N}, \mathrm{N}-1, \mathrm{~N}-2, \ldots, 1$ are lowered in their turn to "uncompute" the calculation of $\mathrm{F}$. We then compute $\mathrm{F}^{-1}$ (output) by raising clock signals $\mathrm{N}+2, \mathrm{~N}+3$, $\mathrm{N}+4, \ldots, \mathrm{N}+\mathrm{M}$. This computation produces exactly the values that are stored in stage 0 , and so we can now unwrite the contents of stage 0 by lowering clock 0 : this turns on the switches connecting the latches of stage 0 to the output of stage $\mathrm{N}+\mathrm{M}$. We then uncompute the calculation of $\mathrm{F}^{-1}$ (output) by succesively lowering clock signals $\mathrm{N}+\mathrm{M}, \mathrm{N}+\mathrm{M}-1, \mathrm{~N}+\mathrm{M}-2, \ldots$, and $\mathrm{N}+2$. Finally, we again raise clock 0 to again isolate the input (which is now all 0 ).

At the end of this process the outputs are stored in stage $\mathrm{N}+1$ and the input is all 0 . The entire pro- 
cess was carried out in a fully reversible manner, and so energy dissipation can in principle be made arbitrarily small. The only thing we need finally do is exchange the data in the input and output latches (we assume that the input and output latches have the same number of bits). To do this, we first transfer the data in the output latches into the input latches and then "unwrite" the output latches. While the circuitry for this is not entirely trivial, it should be clear that the principles developed should allow us to do this. The sequence of steps involved is gone over in detail in the following several paragraphs.

To exchange the data between the input latches and the output latches, we require four additional sets of switches and four additional sets of clock lines. We illustrate the process by considering the exchange between a single input latch and a single output latch. The four new switches are designated simply $\mathrm{S} 1, \mathrm{~S} 2, \mathrm{~S} 3$, and $\mathrm{S} 4$, while the four new clock lines used in the exchange are designated EC1, EC2, EC3 and EC4 (the EC standing for Exchange Clock). Figure 8 shows the arrangement of switches and clock lines. This method of exchanging the data between two latches is similar to the method described by Hall[21].

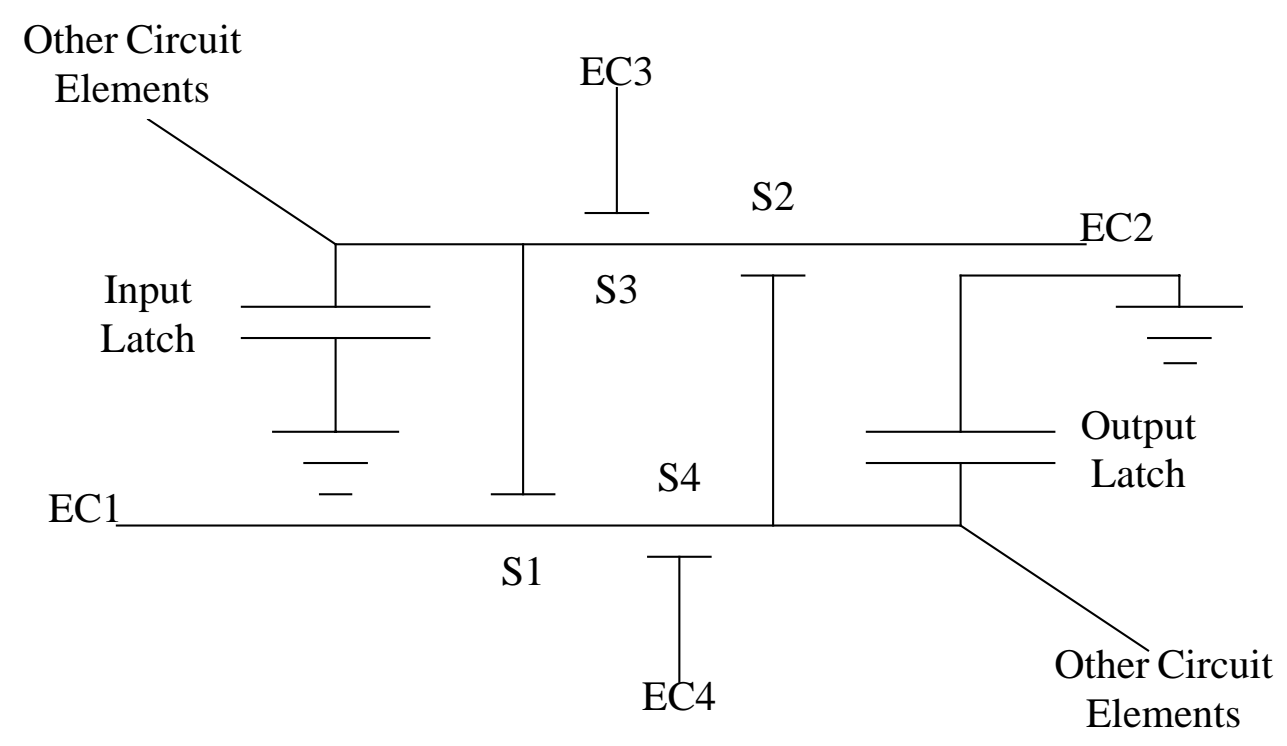

Figure 8

Exchanging Data

Between Two Latches

Initially, EC1 and EC2 are set to 0 while EC3 and EC4 are set to 1. The switches S3 and S4 are turned off. S1 is turned on, because the input latch holds a 0 . S2 could be either on or off, depending on the data on the output latch. We first transfer data from the output latch to the input latch. EC3 is lowered turning on S3. Because the input latch holds 0 and EC2 is set to 0, turning on S3 is permitted. EC2 is then raised to 1 . If $\mathrm{S} 2$ is on, charge is transferred to the input latch. If $\mathrm{S} 2$ is 
off, charge is not transferred. Thus, if the output latch holds a 1 the input latch will be set to 0 . If the output latch holds a 0 , the input latch will be set to 1 . In the process of transferring the data from the output latch to the input latch, we have also inverted it. To correct this omission would require an inverter in the diagram of figure 8 , or some modifications to $\mathrm{F}$ and $\mathrm{F}^{-1}$. This would take an additional switch and an additional clock line, as well as additional explanation. The figure, however, would rapidly become cluttered so we have chosen to omit the inverter to clarify the exposition. This ommision has no impact on the basic concept.

Having set the input latch with data from the output latch, we now raise EC3 and turn off S3. Finally, we lower EC2. This completes the transfer of data from the output latches to the input latches. We must now unwrite the output latches.

Unwriting the output latches is similar to setting the input latches, but the exact sequence of operations is changed. Our first step is to raise EC1. We then lower EC4 turning on S4. EC1 is high, so if there is charge on the output latch then we have turned on a switch when there is no voltage across it, which is permitted. If there is no charge on the output latch, then there will be charge on the input latch (because we transferred the complement of the output latch to the input latch in the earlier steps) and this charge will turn off S1. Therefore, the output latch and EC1 will not be connected. Again, we have not turned on a switch with a voltage across it.

At this point, if the output latch is charged it is connected to EC1. By lowering EC1 we can drain the charge from the output latch. We then raise EC4 turning off S4. This isolates the output latch again and completes the unwriting of the output latch from the input latch.

The clocking sequence, in brief, is: lower EC3, raise EC2, raise EC3, lower EC2, raise EC1, lower EC4, lower EC1, raise EC4.

This sequence of operations has exchanged the data on the input latch and the output latch, and did not require turning on a switch when there was a voltage across it. The careful reader might wonder about the charge that might be trapped on the segment between S1 and S4 or between S2 and S3. These segments are initially 0 , and are restored to 0 upon completion of all operations. During the actual transfer and unwriting steps, the charge on these segments is always known and does not result in turning on a switch with a voltage across it. An explicit capacitance attached to this segment would not alter the conclusions reached here for this specific design. In other designs it might be useful to eliminate such segments. Whether or not such segments can be eliminated depends on the specific implementation. Elimination can often be done by appropriate physical design of the switches, effectively resulting in a single switch with two control inputs.

We have now completed the full cycle of operations required to compute $\mathrm{F}$ (input). We can go on to compute $\mathrm{F}(\mathrm{F}$ (input)), $\mathrm{F}(\mathrm{F}(\mathrm{F}$ (input))), etc. etc. We assume $\mathrm{F}$ is computationally useful (e.g., it implements a single instruction or step of a reversible computation). As discussed earlier, reversible functions can be used to carry out arbitrary computations.

\section{Problems with Inaccurate Voltage}

While the system described is reversible in principle, the energy dissipation in a practical implementation would depend upon the accuracy of the voltage. When a switch is turned on, the logical 
state of the input and output values is the same, but the physical state might differ. A small difference in the voltages at the input and output of the switch would result in energy loss as the switch was turned on. Even with this restriction, it should be possible to achieve significant reductions in energy dissipation. The energy dissipated by dissipatively discharging a capacitive load is

$$
\text { Energy }=\frac{1}{2} C V^{2}
$$

where $\mathrm{C}$ is the capacitance and $\mathrm{V}$ is the voltage. In conventional (irreversible) logic systems, the voltage $\mathrm{V}$ would be the voltage of the power supply. In a reversible system of the type described here the voltage $\mathrm{V}$ would be the error in the voltage of the power supply, rather than the voltage of the power supply itself. Thus, by using reversible instead of irreversible logic, we have effectively reduced the value of $\mathrm{V}$. With a power supply accurate to 5\%, the energy losses from charging and discharging the load capacitances associated with the electrical connections would be decreased by a factor of $20^{2}$ or 400 . That is, energy losses would be a function of the error in the voltage of the power supply, rather than a direct function of the voltage of the power supply itself. There does not appear to be any reason in principle why the error in the voltage of the power supply could not be made as small as desired.

A more fundamental reason for long term optimism is the observation that electric charge is quantized. In future systems in which the number of electrons that represents a logic " 0 " or " 1 " is both finite and small, it should be possible to reduce the energy loss caused by slight inaccuracies in the voltage supply to 0 . It is already possible to control the passage of individual electrons in a circuit $[4,26,69]$. Such highly precise control should eventually make a fully reversible electronic switch with extremely small energy losses feasible.

\section{Problems with FETS: Voltage Degradation}

Switching elements that do not degrade the voltage of the signal being switched are feasible in principle, as shown by the electrostatic relay illustrated in figure 1. CMOS transmission gates can also be used as switches without concern about voltage degradation. Transmission gates require that both the control signal and its inverse be provided. A simple method of satisfying this requirement would be to use two-rail logic. To use nMOS (or pMOS) by itself, we must overcome certain shortcomings. In particular an nMOS switch, when used in pass logic, will cause voltage degradation. As a consequence, deterioration of the voltage through multiple logic levels would impose a severe limit on the capabilities of the system.

Boosting voltage in a fully reversible fashion is, however, straightforwards in principle. The voltage on a capacitor will increase if the capacitance decreases. Variable capacitors are well known based both on physical motion of the capacitor plates, or on changes in charge distribution secondary to a voltage change. Varactors in which the capacitance $\mathrm{C}$ changes by a factor of 10 or more in response to a voltage change of little more than a volt are feasible [25, 30]. The approach outlined here is abstractly similar to the proposal by Joynson et. al.[28].

Boosting voltage is illustrated in figure 9 . Before starting, the switch is turned on and current can flow onto the varactor. The high-voltage clock then opens the switch, trapping the charge on the 


\section{HIGH VOLTAGE \\ CLOCK}

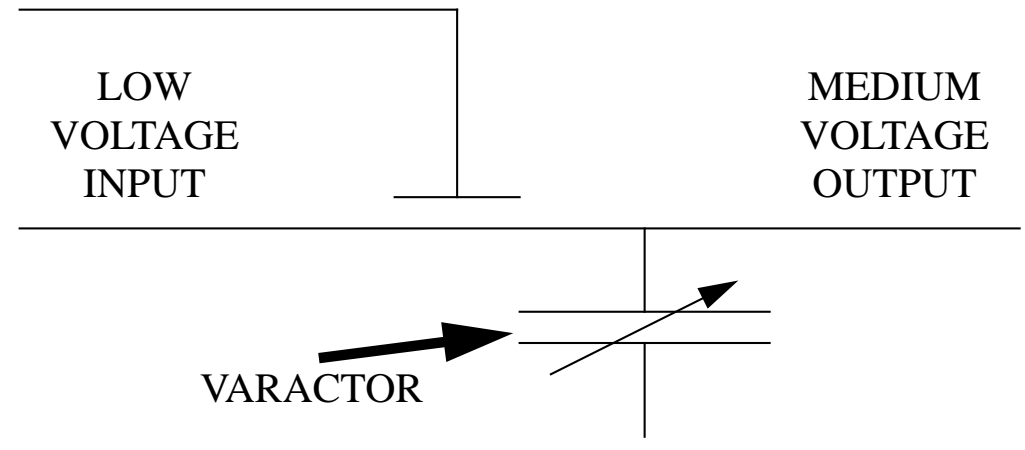

Figure 9. A Reversible

Voltage Booster

varactor. Finally, the capacitance of the varactor is reduced. Because the voltage on a capacitor is

$$
V=\frac{Q}{C}
$$

(where $\mathrm{V}$ is voltage, $\mathrm{Q}$ is charge and $\mathrm{C}$ is capacitance) the voltage across the varactor will increase as its capacitance decreases. Because the voltage on the high-voltage clock is large, there is more than sufficient control voltage to insure that the switch remains turned off even though the voltage across the switch has been increased. The low-voltage input, boosted to a medium-voltage output, can then be used to switch a low-voltage signal, which can again be used as the input to a reversible voltage booster.

To reverse this cycle of operations, the capacitance of the varactor is returned to its original value. This will also return the voltage on the output to its original value, and the high-voltage switch can be turned on with minimal energy loss. This energy loss will be a function of the square of the error in the voltage regulation. If the number of electrons that charge the varactor (and any stray output capacitance) is reduced to a small integer number, it should eventually be possible to eliminate losses due to small inaccuracies in voltage regulation.

Seitz et. al. [29] commented that "...the isolation transistor can turn on while there is voltage across it, and accordingly, it dissipates power in charging or discharging the bootstrap node. The goal of exporting all of the dynamic power is elusive." Translating from their language to the language used here, they sometimes allowed the high-voltage clock to turn on the high-voltage switch when a voltage existed across the switch, thus dissipating energy. They did not consider the computation of $\mathrm{F}^{-1}$ and "unwriting" operations, and so were sometimes obliged to engage in the dissipative operation of discharging the gate of a FET to ground. In essence, they were able to eliminate unnecessarily dissipative operations for combinational logic, but not for sequential logic. As shown here, appropriate design of a reversible sequential circuit eliminates the unnecessarily dissipative steps. 


\section{Problems with FETS: Leakage Current}

Another problem with FET's is leakage current. Even a FET in which the gate voltage is sufficient to insure that the FET is completely cut off will still pass a small current. If our objective is to achieve energy dissipations below $k T$, any significant leakage current is intolerable.

Leakage current occurs because thermally generated electron-hole pairs are created in the semiconductor material of the FET. Thermal agitation will occasionally cause an electron in the valence band (e.g., an electron which normally would not be able to move because it was fixed in place as part of a covalent bond) to leap into the conduction band. An electron in the conduction band is free to move throughout the crystal and so to conduct electric current. The energy gap between the valence band and the conduction band will govern the concentration of electrons that are available to carry leakage current. In silicon, the bandgap is 1.12 electron volts. At 300 kelvins, this will produce $1.45 \times 10^{10}$ electrons per cubic centimeter of silicon. This "intrinsic carrier concentration" will result in dissipative energy losses.

Three obvious methods are available for reducing the leakage current:

(1) Reduce the physical size of the device. An intrinsic carrier concentration of $1.45 \times 10^{10}$ electrons per cubic centimeter can also be expressed as $1.45 \times 10^{-11}$ electrons per cubic nanometer. If the active region of the device was smaller than a few millions of cubic nanometers, then the probability of finding an undesired charge carrier in that region would be relatively small.

(2) Reduce the temperature. The intrinsic carrier concentration is:

$$
n_{i}=k_{m} e^{\frac{-E_{g}}{2 k T}}
$$

where $n_{i}$ is the intrinsic carrier concentration, $k_{m}$ is a constant specific to the material, and $E_{g}$ is the bandgap energy. In the case of silicon, $k_{m}$ is about $3.1 \times 10^{20}$. At a temperature of 77 Kelvins, $n_{i}$ is $7.4 \times 10^{-17}$ electrons per cubic centimeter. CCD's with dark currents of a few electrons per pixel per hour have been fabricated[51].

(3) Select a material with a larger bandgap. Diamond has a bandgap of 5.47 electron volts, with an intrinsic carrier concentration at room temperature in the range of $10^{-26}$ electrons per cubic centimeter. Boron provides a good shallow dopant, and p-type diamond has been made. Several shallow dopants for $n$-type material are plausible[20]. Diamond MOSFETs with boron doping have been fabricated[58]. An implementation could use purely p-type materials, if that proved useful. A variety of wide band-gap materials exist allowing a wide range of tradeoffs between energy dissipation and other parameters.

It should be feasible to produce acceptable leakage currents using a variety of different materials in a variety of different geometries under a broad range of operating temperatures. The use of small FET's using a wide band-gap material is particularly attractive for room temperature operation. In such a structure the probability of finding even one valence electron in the conduction band is astronomically small. 
Thermally generated leakage current is also of concern in many types of varactors. A reverse-biased $\mathrm{P}-\mathrm{N}$ junction illustrates the problem: electron-hole pairs generated thermally in the charge depletion region are swept out of the region, thus conducting current. Leakage current in such varactors can in principle be made quite small for the same reasons that apply to leakage current in a FET.

\section{Reversible CCD-Based Logic}

An alternative approach, which eliminates the need for high accuracy voltage supplies, is to eliminate the need to turn on the switch when there are charge carriers present. This might be viewed as only turning the switch on and off when the voltage across the switch is 0 volts, but this is not a fully adequate description. Even a 0 volt signal is subject to fluctuation and noise. If, however, we try to put a positive voltage onto the switch (e.g., the inputs and outputs of the switch both have a positive voltage), and if the charge carriers in the switch are electrons, then it is possible to remove all the charge carriers from the channel region. Any charge carriers will be actively swept out of the channel region by the positive voltages on the input and output leads. Once all the charge carriers have been removed from the channel region, then no more current will flow. Precise regulation of the positive voltage on the input and output of the switch is not required, for both need merely be "sufficiently positive" to insure removal of all charge carriers from the channel region. Once the charge carriers have been removed then turning the switch on and off can be done in a fully reversible manner. Small errors in the supply voltage can no longer lead to dissipative current flows when the switch is turned on, because no current can flow.

For purposes of this discussion we will assume our switch is a FET with electrons as the charge carriers and that the electrons are removed from the FET by a positive voltage on the input and output leads. (We could equally well have assumed the charge carriers are holes and that a negative voltage is placed on the input and output leads: for purposes of explanation the choice is arbitrary). As discussed earlier, it is essential that the device be designed so that the concentration of intrinsic charge carriers is sufficiently low that energy losses from this source can be neglected. Further, the density of device defects must be low enough that defect sites do not appreciably alter device performance. We will simply assume that the material is free of defects. Reduction of device size coupled with decreases in defect rates should eventually allow construction of devices with a sufficiently low defect rate that energy dissipation will not be significantly degraded.

In this approach, 1's and 0's are represented by a packet of electrons or the absence of a packet of electrons, respectively. The electrons are stored in potential wells which we will call "buckets." Each packet of electrons is treated as a unit: we never split or merge packets, nor do we create or destroy packets (except when the system is initialized, which would ideally occur only once). While all packets have approximately the same number of electrons, it is not critical that each packet have exactly the right number of electrons. Whether a packet has 99, 100, or 101 electrons won't matter.

The use of charge packets is most familiar in the context of CCDs (Charge Coupled Devices)[47, 50], or alternatively in the context of BBD's, or Bucket Brigade Devices[49]. In a CCD, charge packets are transferred from potential well to potential well serially along a row of devices. The amount of charge in each packet is a measure of some analog quantity. Such devices are primarily used as memory elements or shift registers, not for computation. Proposals to use the same basic 
concepts for logic operations have been made since the invention of the $\operatorname{CDD}[34,35,36,46,48]$. Sometimes called "DCCL," or Digital Charge Coupled Logic, these proposals have explored the advantages of using charge packets as the basic logic element. The primitive logic elements in prior proposals, however, were highly dissipative. For example, Zimmerman et. al.[34] proposed logic operations whose computation required merging packets, discarding packets by draining the charge to ground, etc. The goal of thermodynamically reversible logic was not an objective. Tompsett[48] proposed circuits in which the potential generated by a charge packet in a CCD potential well was coupled to the gate of a FET. This FET would then be turned on or off depending on whether a charge packet was present or absent in the potential well. This was then used to create another charge packet (rather than to control the movement of an existing charge packet). The creation of a new charge packet would compensate for imperfect charge transfer. NAND and NOR operations could be performed by applying the potential from two potential wells to two gates of two FETs, which could then be connected in serial or parallel. Again, the objective of thermodynamic reversibility was not considered. The method of sensing charge in the potential well was dissipative and the sensed packets, no longer needed, were to be dissipatively discarded.

For simplicity, we will assume that packets never gain or lose electrons (due to defects in the material or to thermal noise). In fact, present day devices can gain or lose charge at the rate of a few parts per million[51]. As discussed earlier, such gains or losses should eventually be reduced to insignificant levels. Even if we allow packets to gain or lose electrons at some low rate, we could periodically "refresh" the packets, and so prevent malfunction. Tompsett[48] first proposed methods of refreshing packets (though he did not consider reversible methods). To the extent that the packet has gained or lost an unpredictable amount of charge, "refreshing" the packet must fundamentally dissipate energy. Other than this fundamental loss, however, we wish to keep energy dissipation to a minimum. A reversible method of refreshing a packet would be to use the old packet to charge a bucket in a reversible fashion (as discussed in the first method), and then to "unwrite" or discharge the bucket holding the old packet by using the new packet as the source of information. This method of refreshing a packet would, of course, dissipate energy as a function of the errors in the voltages involved (as discussed earlier). If the refresh operations were done infrequently (e.g., a packet would be refreshed only after a large number of logic operations) then the energy dissipation per logic operation from this source would be quite small.

While CCD and DCCL circuits are today implemented on a semiconductor surface using planar technology, for purposes of this discussion we will initially ignore this geometrical constraint and instead focus on more fundamental issues. A planar version of the ideas described here is given in the section on "A Planar Version of RCT Logic."

Figure 10 illustrates the "hydraulic" model[1] of charge transfer in a CCD. Charge is transferred from one potential well to the next much like water would flow to a gradually sinking region in a pond. This process can be thermodynamically reversible when appropriately implemented.

One aspect of figure 10 might be slightly misleading. While water has a sharply defined surface, the energy of an electron at some non-zero temperature $T$ is statistical. While figure 10 might lead one to conclude that extremely small perturbations in the potential might cause significant changes in the pattern of charge flow, in fact thermal noise will allow electrons to surmount small barriers e.g., where the barriers have a potential similar in magnitude to $k T / e$ or .026 volts at room temperature (where $e$ is the charge on an electron). Small perturbations and errors in the potential do not 

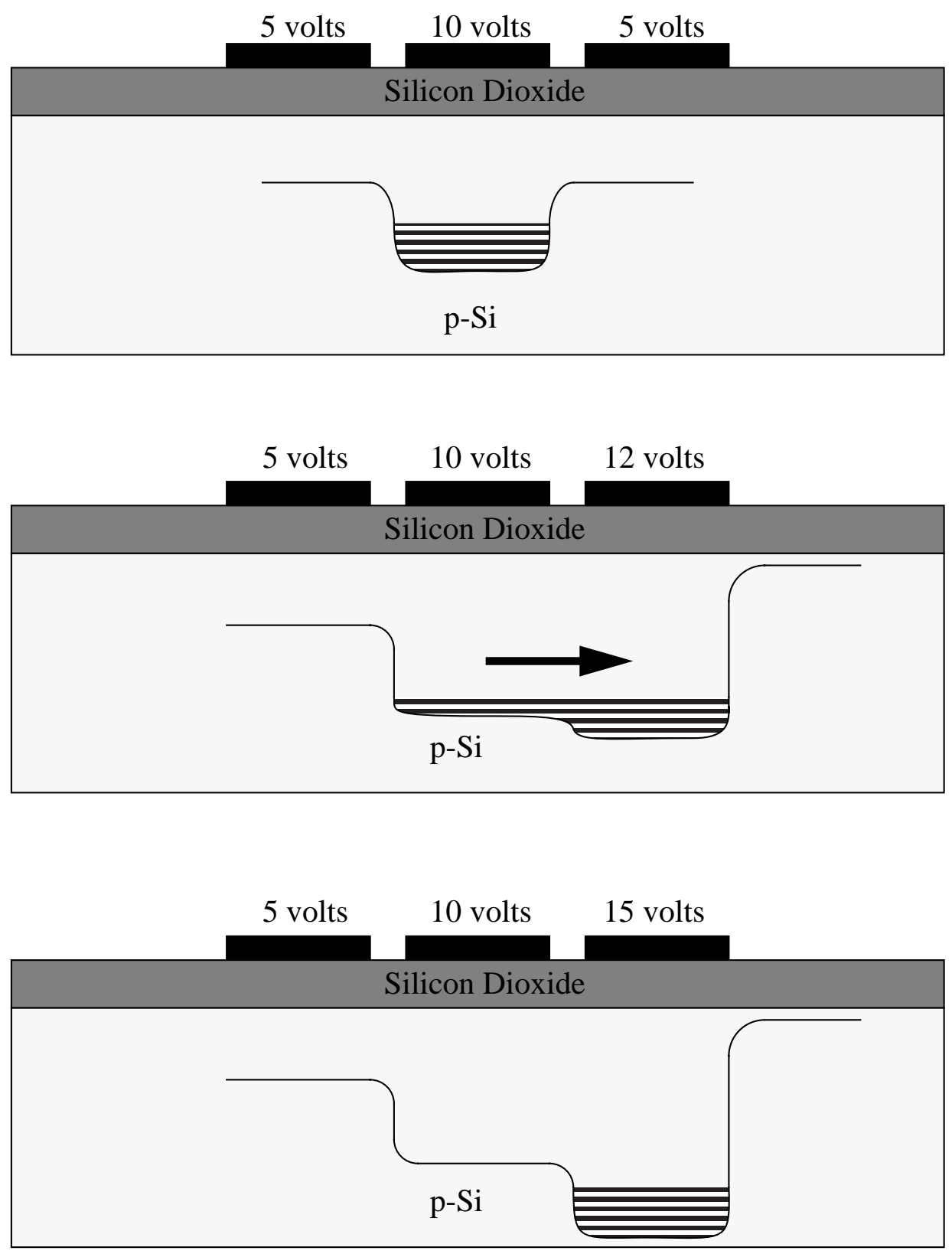

Figure 10

Hydraulic Model of Charge Transfer in a CCD (Adapted from Sze[1])

Negative Charge (electrons)

fundamentally create dissipative problems.

\section{Tranferring Charge With Little Dissipation}

Before discussing the more complex operations needed for computation, we first consider a simple 
sequence that transfers charge from a source bucket to a destination bucket with as little energy loss as desired. This is illustrated in figure 11. (Note that the illustration shows that the two buckets

\section{SOURCE}

\section{DESTINATION}

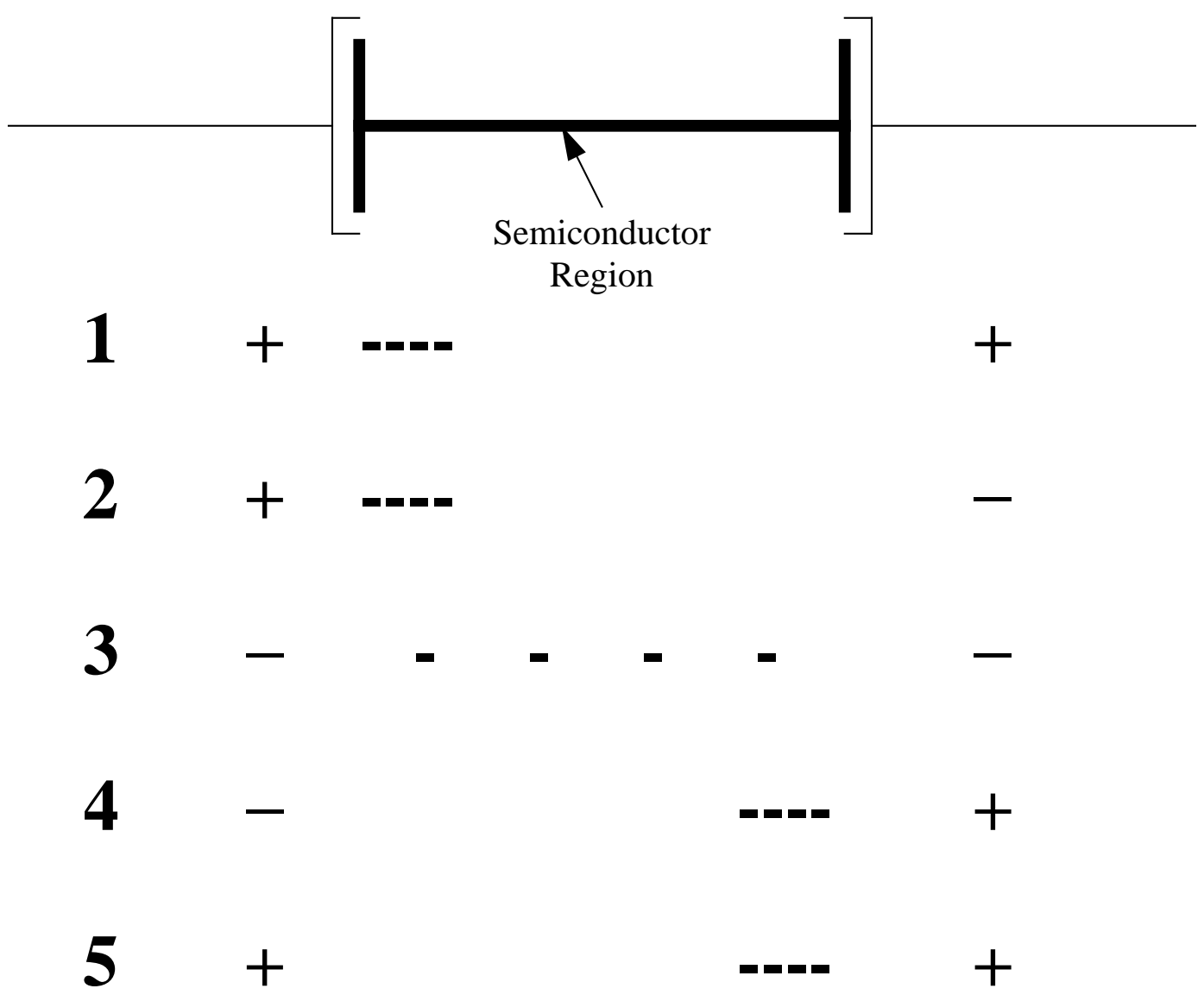

\section{Charge Distribution}

Figure 11

Transferring Charge

With Little Dissipation

have semiconductor plates joined by a semiconductor path. The use of metallic plates or a metallic connection between the two plates would cause unwanted energy dissipation). At first, both the source clock and destination clock are + (positive), and the charge packet is entirely in the source bucket. The clocking sequence will then shift the charge from the source bucket to the destination 
bucket in a series of steps, where each step can dissipate an arbitrarily small amount of energy. As we move from state 1 to state 2 , the destination clock is changed from + to - . This change results in negligible energy dissipation, because no charge can move at this time. As we move from state 2 to state 3 , the source clock is changed from + to - The charge carriers in the charge packet (electrons in our example) now move out of the source bucket. Because the destination clock is negative, the electrons will migrate smoothly out of the source bucket. (If the destination clock were + at this point instead of -, the electrons would move out of the source bucket and then "fall down hill" into the destination bucket, dissipating energy). As we move from state 3 to state 4 , the destination clock changes from - to + , thus slowly attracting the electrons and gathering them into the destination bucket. Finally, as we move from state 4 to state 5, the source clock changes from - to + restoring the system to its original condition, but with the charge packet in the destination bucket instead of the source bucket.

The primary source of energy dissipation in this sequence is from state 2 to state 3 , and from state 3 to state 4 . These transitions involve charge migration, and will therefore result in dissipative losses. As we slow the clock frequency the clock voltages change more slowly, the charges move more slowly, and hence dissipate less energy. Because energy dissipation is a function of the square of the current, reducing the frequency of operation by a factor of two will also reduce the current by a factor of two but will reduce the energy dissipation by a factor of 4 . This will reduce the energy dissipated per logic operation by a factor of 2 . Thus, by sufficiently slowing the clock frequency we can dissipate as little energy per operation as might be desired.

It is important to notice that these operations do not erase information. We start with a charge packet in a single potential well on the left, and we end with a charge packet in a single potential well on the right. During the course of moving the charge packet we slowly change the shape and position of the potential well, but at no point do we merge potential wells. The potential well at the right is initially empty, which is why the transition from step 1 to step 2 in figure 11 is allowed and nondissipative. This transition eliminates the right hand potential well without merging it with the well on the left. An attempt to merge the two potential wells would be dissipative. The transition from step 2 to step 3 slowly changes the shape of the potential well, but the charge packet is always contained in a single potential well and is always at or near thermodynamic equilibrium within that well.

\section{The Primitive Operation}

We now consider the somewhat more complex operation that is required to support computation in a reversible fashion. The single primitive operation is:

Transfer charge out of a source bucket and into an empty destination bucket if a "condition" bucket holds a " 0 ", but do not transfer the charge if the condition bucket holds a " 1 ". If the transfer does not take place (the condition bucket is "1"), the destination bucket need not be empty.

"Empty" in this context means "has no charge carriers present." That is, an "empty" bucket is in logic state "0."

We will call this device a 3-bucket Reversible Charge Transfer device, or (where context makes it clear which type of RCT we are referring to) simply an RCT. The RCT is a reversible DCCL de- 
vice. At a logical level the RCT is related to (but somewhat different from) a switch gate[7, page 241] implemented using charge packets instead of colliding billiard balls. The particular logic function described here is but one example of the range of logic functions that involve the reversible transfer of charge from one bucket to another. During normal operation, RCT devices (1) never merge or split charge packets (2) never discharge charge packets to ground, and (3) move charge packets in a thermodynamically reversible fashion from a set of source buckets to a set of destination buckets, conditional on the presence or absence of charge in a set of condition buckets. In this context thermodynamic reversibility means that energy dissipation per device operation, in the absence of manufacturing defects, could be made much smaller than $k T$ simply by slowing the speed of operation. The number of charge packets is conserved and therefore RCT devices are conservative logic devices (in conservative logic devices, the number of 1's is conserved during the computation, so the number of 1's at the input is the same as the number of 1's at the output). We have described the 3-bucket RCT, which has one condition bucket, one source bucket, and one destination bucket. More complex RCT's have more buckets and the pattern of charge transfer is more complex. Some of these RCT's will be considered later.

The simple 3-bucket RCT operation might be written in $\mathrm{C}$ as:

$$
\text { if }(\text { Condition }==0)\{\text { Destination }=\text { Source } \text { Source }=0\} \text {; }
$$

while a somewhat briefer notation would be:

$$
\text { If Condition is } 0 \text { then Destination }=\text { Source. }
$$

(Note that in this briefer notation, the "=" sign is used to indicate both the assignment of the Source value to the Destination, and setting the Source to 0 after the assignment).

The precondition that must be true prior to execution of the 3-bucket RCT is:

$$
(\text { Destination==0) OR (Condition==1) }
$$

Violation of this precondition would result in an unwanted dissipative step, which is banned in RCT devices. While it might at first seem that satisyfing this precondition would make the 3-bucket RCT data dependent, it is in fact possible to design a circuit in which we know that the precondition is satisfied without knowing what values are presented as inputs to the circuit.

A logic diagram showing the possible initial and final states of a 3-bucket RCT is shown in Figure 12. On the left, the legal initial states of the RCT are shown, while on the right the final states (after the sequence of clock signals that cause the device to step through a single cycle of operations) are shown. It is interesting to note that there is only a single legal, non-trivial change of state: when the input is 1 , the condition is 0 , and the output is 0 . In all other cases either the input and output states are the same or the input state is illegal. The illegal states would cause undesired energy dissipation.

\section{Implementing a Fredkin Gate with the 3-bucket RCT}

The 3-bucket RCT is logically complete, for we can implement a Fredkin gate with it. The con- 
Initial State

Source Condition Destination Source Condition Destination

Figure 12

Allowed states and state changes of a 3-bucket RCT device. Double headed arrow denotes the only legal, nontrivial change of state.

struction used here is related to (though diffrent from) that used by Fredkin and Toffoli[7] for constructing a billiard ball Fredkin gate from a billiard ball switch gate.

A Fredkin gate has three inputs and three outputs. The inputs are the control input $\mathrm{A}$, and two signal inputs B and C. The outputs are $\mathrm{A}^{\prime}, \mathrm{B}^{\prime}$, and $\mathrm{C}^{\prime}$. If the control input $\mathrm{A}$ is 0 , then $\mathrm{A}^{\prime}=\mathrm{A}, \mathrm{B}^{\prime}=$ $B$, and $C^{\prime}=C$. If the control input $A$ is 1 , then $A^{\prime}=A, B^{\prime}=C$, and $C^{\prime}=B$. That is, the output of the Fredkin gate is identical to the input if $A=0$, but $\mathrm{B}^{\prime}$ and $\mathrm{C}^{\prime}$ are exchanged if $\mathrm{A}=1$.

The Fredkin gate is illustrated in Figure 13.

The following sequence of simple RCT operations will implement a Fredkin gate:

$\mathrm{A}, \mathrm{B}$, and C hold arbitrary input values. InitiallyOne is set to a logic "1" (the presence of an electron packet). All other variables are initialized to logical "0" (the absence of an electron packet).

1.) If $A$ is 0 then $C^{\prime}=C$.

2.) If $\mathrm{A}$ is 0 then $\mathrm{B}^{\prime}=\mathrm{B}$.

3.) If $A$ is 0 then $\operatorname{Not} A=$ InitiallyOne.

4.) If Not $A$ is 0 then $C^{\prime}=B$.

5.) If Not $A$ is 0 then $B^{\prime}=C$.

6.) If AlwaysZero is 0 then $A^{\prime}=A$.

7.) If $A^{\prime}$ is 0 then InitiallyOne $=$ NotA. 

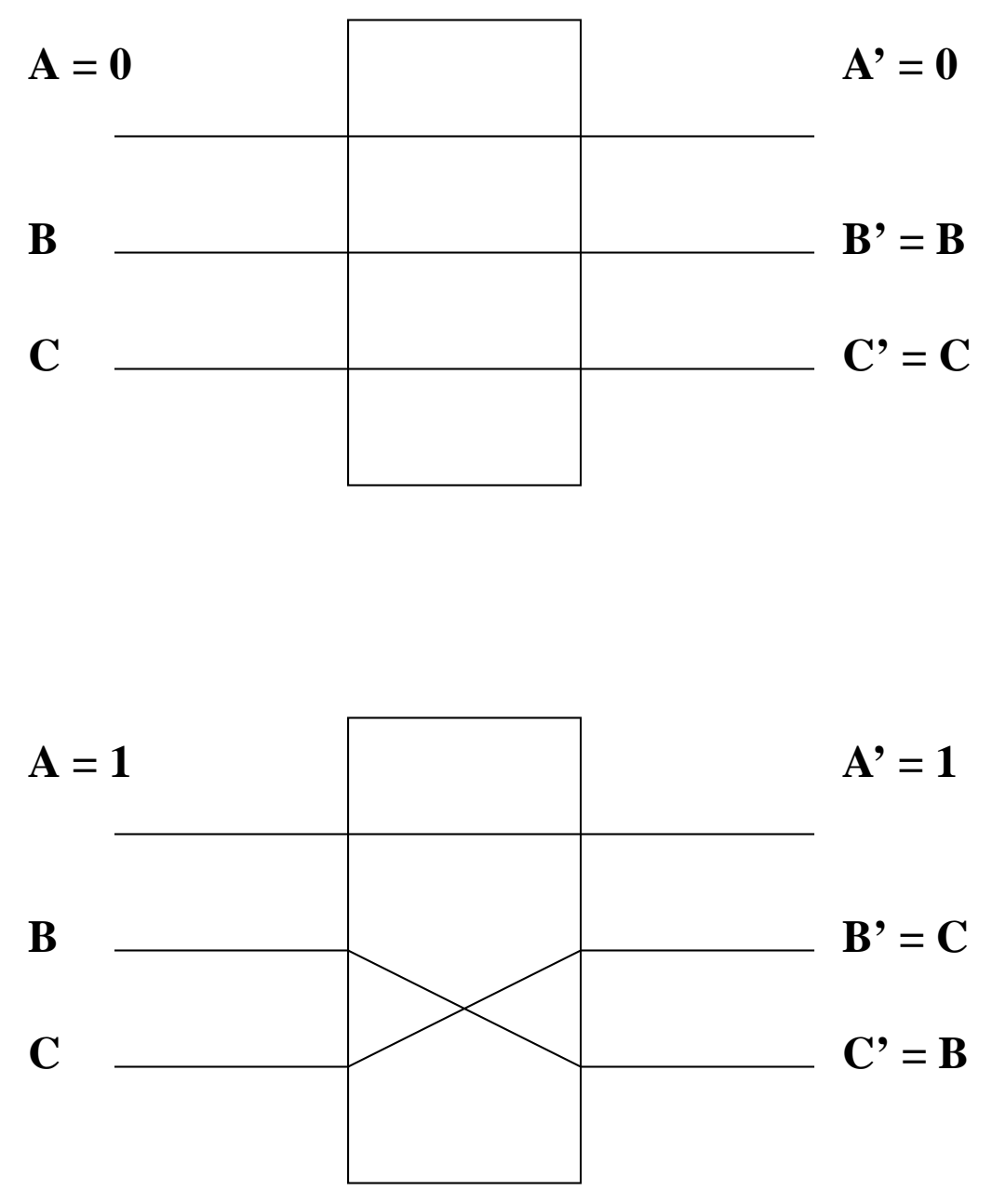

Figure 13

A Fredkin Gate

Steps 1 and 2 simply transfer charge from B and C into B' and C' if A is 0 . B' and C' are initially empty, so this transfer satisfies the RCT preconditions.

Step 3 computes the logical negation of A and leaves that logical negation in "NotA."

Steps 4 and 5 transfer charge from $B$ and $C$ into $C^{\prime}$ and $B$ ', exchanging the outputs. The precondition is satisfied, for if $\mathrm{A}$ is 0 , then NotA will be 1 and the transfer will not take place. If the transfer does not take place, the prior contents of $\mathrm{B}^{\prime}$ and $\mathrm{C}^{\prime}$ are irrelevant. If $\mathrm{A}$ is 1 , then NotA will be 0 and the transfer will take place. In this case, B' and C' will be empty (have logic values 0 ) and so the transfer will take place correctly. 
Step 6 copies A into A' unconditionally.

Step 7 restores the value of InitiallyOne if it was altered during the computation.

This implementation of a Fredkin gate will take three arbitrary input values and, after a sequence of RCT operations, produce three output values. We could clearly apply N Fredkin gates to $3 \mathrm{~N}$ input values and produce $3 \mathrm{~N}$ output values at the same time. We require that all output values be initially 0 . After finishing the sequence of clocking operations, the inputs are all 0 and the outputs hold arbitrary values. At this point, the "input" and "output" can be logically exchanged (e.g., no physical operation takes place, but we relabel the "input" variables as "output," and the "output" variables as "input"). Following this logical exchange, we have reestablished the preconditions for the next sequence of operations: the (just relabeled) inputs again hold arbitrary logic values, while the (just relabeled) outputs hold logic 0's. We now drive a (different) set of clock lines to apply the (different) Fredkin gates to generate the next "state" of the computation.

Viewed globally, this is a "ping-pong" or "double buffer" scheme for computing successive values of the global state of the system. If we call the two sets of variables $A$ and $B$, and we call the two logic functions for the "next state" of the system F and G, then we first compute B=F(A). B is initially all zeroes, while A holds arbitrary logic values. On completion of the computation of $F, A$ is all zeroes while $B$ holds arbitrary logic values. We then compute $A=G(B)$. Because $G$ uses $B$ as its input and $A$ as its output, the condition that the outputs be all zeroes is met during the compuation of G. This returns us to the state where A holds arbitrary logic values and B holds all zeroes. We can repeat this cycle indefinitely.

\section{Implementation of a 3-bucket RCT}

A planar layout of a 3-bucket RCT is shown in figure 14. Many kinds of implementations would be feasible, for example Silicon-On-Insulator technology (the FET used for the transfer switch would be "tilted on its side" in this approach). Note that many of the conductive paths are really semiconductors. Each bucket also has one semiconductor plate. The gate is also a semiconductor. Use of metallic wires for these components would compromise device function. A planar RCT is described in a later section.

This RCT can be viewed as a combination of three CCD potential wells and a FET. A charge packet is first transferred from the "condition" potential well to the gate of the FET. The charge packet in the "source" potential well is then moved to the source of the FET and (the charge on the gate of the FET permitting) through the channel and out the drain of the FET into the "destination" potential well.

Tompsett[48] proposed sensing the presence or absence of a charge in a CCD potential well by connecting the potential well via a doped region connected ohmically to a metalization that became the gate electrode of a FET. Charge injection from the gate into the doped region, while small if the capacitance of the gate is small, still introduces an unwanted source of energy dissipation. Other aspects of Tompsett's proposal were also irreversible or highly dissipative. In the present proposal, we require that both the gate of the FET and the connecting path from the potential well to 


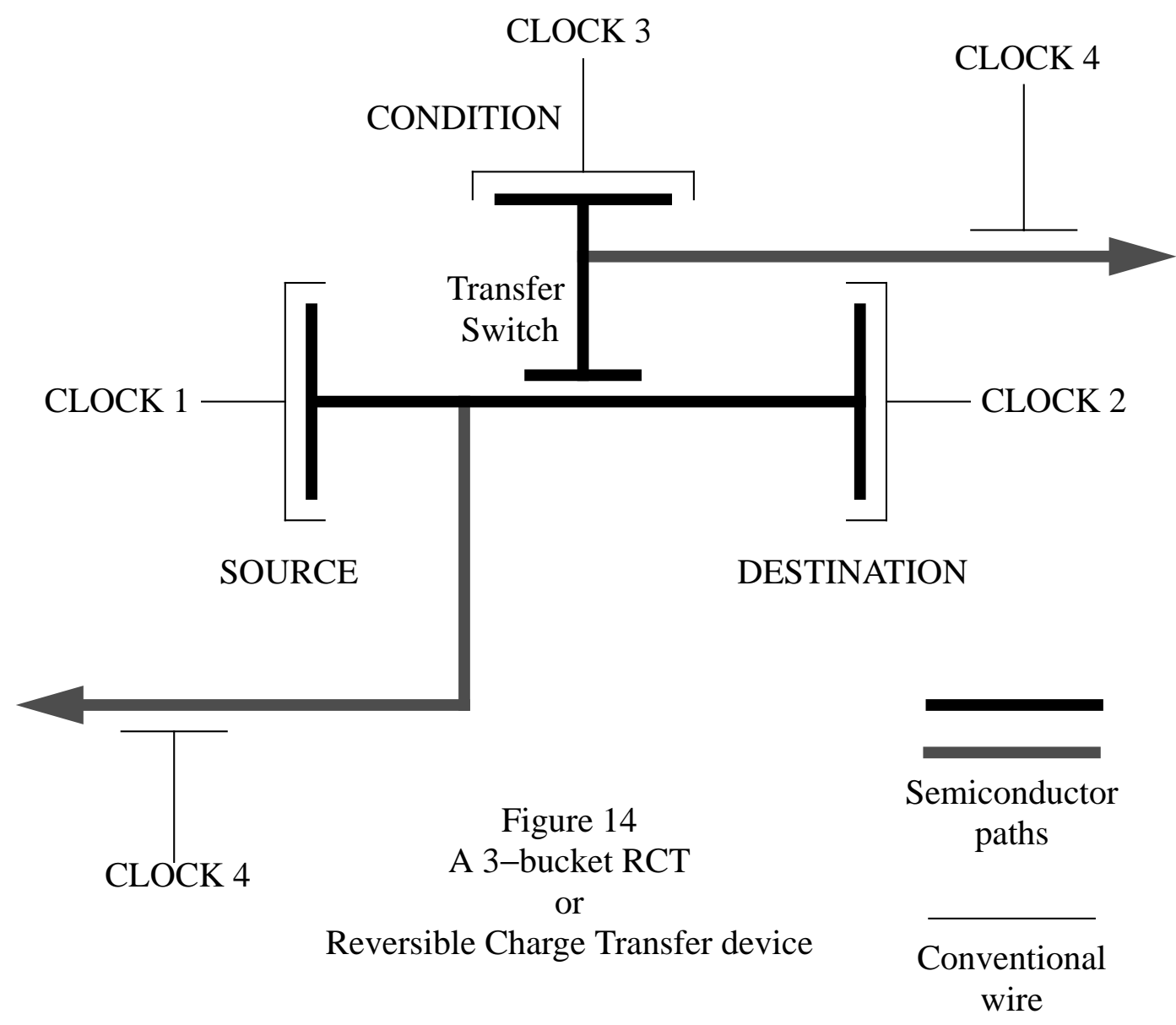

the gate be a semiconductor. The only charge carriers in this semiconductor path would be those deliberately introduced as a charge packet. An alternative approach which might be more convenient in a simple planar implementation would be to introduce a capacitive coupling into the path from the potential well to the gate. The gate could then be metallic, one plate of the capacitor (the plate connected to the gate) could also be metallic, but the other plate and the connection to the potential well could be a semiconductor. Potential could be coupled through the capacitor, but charge carriers would be prevented from moving into or out of the semiconductive region and hence charge injection from the gate electrode would not be a problem.

Initially, a charge of unknown value is present in the source bucket. The destination bucket is either empty (if the condition bucket holds a 0 ) or has an unknown charge (if the condition bucket holds a 1). Clocks 1, 2, and 3 are all positive. All charge carriers (electrons) have been drawn onto the semiconductor plates of the buckets. The conventional plates are connected to the clocks. There is a net positive charge on each bucket creating a potential well which holds the electrons in the bucket and prevents them from migrating away. The channel regions of all switches have no charge carriers present.

Clock 4 is driven negative with a large voltage. This large voltage cuts off and isolates the three buckets involved from any other buckets to which they might normally be connected. Additional switches controlled by clock 4 might be required to further isolate the three buckets in a larger cir- 
cuit, but are not shown in this small example. The two gray channels do not actively participate in the process of reversible charge transfer, they are present merely to show that connections to other circuit components will be present in a real system.

Clock 3 is driven negative, forcing charge from the condition bucket onto the gate of the transfer switch. If no charge is present on the condition bucket, no charge will be put onto the transfer gate, while if charge is present then it will be transferred to the transfer gate, cutting off the transfer switch. Note that the circuit is so arranged that only a medium voltage will be developed on the transfer gate, and this medium voltage can be contained by the high voltage of the cut off clock, clock 4.

Clock 2 is driven negative. If there is charge on the destination bucket, then the transfer switch is turned off and the charge on the destination bucket goes nowhere. If there is no charge on the destination bucket, then whether the transfer switch is turned off or on is immaterial, for no charge will be transferred.

Clock 1 is driven negative. If the transfer switch is turned off, no charge will be transferred. If the transfer switch is turned on, charge will move gradually through the transfer switch into the destination bucket. Because clock 2 is negative, charge will not "fall down hill" in a dissipative fashion when moving into the destination bucket. Clock 1 has a low voltage so a low voltage is generated during the transfer operation. The medium voltage on the transfer gate is sufficient to block charge transfer if charge transfer is not supposed to take place.

Clock 2 is driven positive, allowing charge to flow smoothly into the destination bucket. When clock 2 reaches its full positive value, all the charge is held on the destination bucket and no charge carriers are present in either the source bucket or the transfer switch channel region (if the transfer switch was turned on).

Clock 1 is driven positive, which has no effect if the transfer took place and simply allows the charge to return to its original state if the transfer did not take place.

Clock 3 is driven positive, allowing the charge on the transfer gate to return to the condition bucket.

Clock 4 is driven positive, opening the cut off switches that isolated the three buckets from outside influence during the reversible transfer operation.

This cycle of operations reversibly transfers charge from the source bucket to the destination bucket if the condition bucket holds a logic "0" (e.g., no charge).

\section{Energy Loss}

There are losses during the RCT cycle of operations caused by current flowing through a resistive medium. The power lost will be

$$
\text { PowerLoss }=I^{2} R
$$

where $\mathrm{I}$ is the current and $\mathrm{R}$ the resistance. Current is proportional to the frequency of operation of the circuit, so if the frequency is low the current will also be low. Therefore, power losses from 
resistive losses will fall off with the square of the frequency:

$$
\text { PowerLoss } \propto f 2
$$

Because the number of operations per second is also lower at a lower frequency, the actual energy loss per device operation is proportional to the frequency of operation:

$$
\text { EnergyLoss } \propto f
$$

By reducing the frequency of operation, the energy loss per operation can be reduced to whatever extent is desired. It should be remembered that "low frequency" operation is relative: CCD's with 0.9997 charge transfer efficiency operating at 1 gigahertz have been demonstrated[54].

More specifically, the electron drift velocity $\mathrm{v}_{\mathrm{d}}$ equals the electric field $E$ times the mobility $\mu$ :

$$
\mathrm{v}_{\mathrm{d}}=E \mu
$$

If we let $\mathrm{d}$ be the distance traveled by an electron as it moves from one bucket to the next, and $\mathrm{t}$ be the time of a single logic operation, then it is approximately the case that:

$$
\mathrm{d}=\mathrm{v}_{\mathrm{d}} \mathrm{t}
$$

The total energy dissipated by the movement of $n$ electrons as they move from the source to the destination is simply the force times the distance times the number of electrons, or:

$$
\mathrm{E}_{\text {dissipated }}=\text { neEd }
$$

where $\mathrm{E}_{\text {dissipated }}$ is the energy dissipated, $\mathrm{n}$ is the number of electrons in a packet, and $e$ is the charge of an electron.

Putting these together yields:

$$
\mathrm{E}_{\text {dissipated }}=\frac{\mathrm{ned} \mathrm{d}^{2}}{\mu \mathrm{t}}
$$

If we assume that the distance traveled by the charge packet as it moves from one bucket to the next is .1 microns $\left(10^{-5}\right.$ centimeters), that one packet has 100 electrons, that the mobility is $1800 \mathrm{~cm}^{2} / \mathrm{V}-\mathrm{s}$ (the approximate mobility of diamond or silicon near room temperature), that the time allotted for one operation is 1 nanosecond, and the charge $e$ on an electron is $1.6 \times 10^{-19}$ coulombs, then the energy dissipated is approximately $10^{-21}$ joules.

This estimate is based on a classical model and involves some significant simplifications. Most significantly, the mean free path will of necessity be less than .1 micron in a device whose maximum dimension is .1 micron, and so the meaning of the electron mobility $\mu$ is somewhat obscured. A more detailed quantum analysis is essential as the size becomes smaller[44]. A rough approxi- 
mation to such an analysis can be based on the observation that the resistance of the channel of a sufficiently small FET (e.g., one in which the channel width is perhaps a few nanometers and only a single transmission mode exists) is roughly half of $\mathrm{h} / e^{2}$, where $\mathrm{h}$ is Planck's constant. This is a fundamental unit of resistance of about $26 \mathrm{~K} \Omega$. A packet of $n$ electrons flowing in time t produces a current of ne/t. The power produced by such a current flow is $\mathrm{I}^{2} \mathrm{R}$, while the energy dissipation per logic operation is $\mathrm{I}^{2} \mathrm{R} \times \mathrm{t}$, or $(\mathrm{n} e / \mathrm{t})^{2} \mathrm{x} \mathrm{h} /\left(2 e^{2}\right) \times \mathrm{t}$ which simplifies to:

$$
\mathrm{E}_{\text {dissipated }}=\frac{\mathrm{hn}^{2}}{2 \mathrm{t}}
$$

For a switching time $t$ of 1 nanosecond and a packet size $n$ of 100 electrons, this is $3 \times 10^{-21}$ joules for each packet that is passed through the channel of the FET. Under the specific conditions considered here this second approximation agrees at least in order of magnitude with the estimate based on a classical model.

It is interesting to note that the two equations have significant differences. The resistance of the channel in the classical case varies with the length of the channel. The length of the channel is less significant in the nonclassical case. The size of the charge packet, n, also influences energy dissipation in different ways in the two cases. In the classical case, the energy dissipation is a function of $n$ rather than $n^{2}$. In the classical case, two electrons drifting under the influence of the same electric field in separate regions of the channel will dissipate twice as much energy as a single electron. In effect, the resistance has been halved because the number of charge carriers has been doubled. A similar effect would occur in a doped semiconductor if the doping density were doubled. If we assume a narrow channel that can only support a single transmission mode, however, then doubling the size of the charge packet will cause more electrons to move through the same channel in the same time period, with a resulting increase in interactions among the electrons. The reader should note that no claim of universality or high accuracy is implied for either of the above equations. They are rough approximations that apply only under a limited range of conditions. These conditions need not, in general, be true. In particular, it would seem attractive to design devices in which single electrons are always close to their ground state both during charge transport and during switching operations. This would occur if the electron were always confined in a time varying potential well of relatively small dimensions. Devices with this property should be feasible when we are able to manufacture devices of sufficiently small size[67]. This should have a favorable influence on energy dissipation.

The major factor ignored in this analysis is the energy dissipated by the clock signals. Many methods of providing periodic clocking pulses are possible. Three methods are discussed in the next section.

The mobility can be substantially increased by reducing the temperature. Mobilities greater than 1,000,000 have been demonstrated in GaAs-AlGaAs HFET's at around 10 K[1, page 298]. While the impact of this is unclear when the device size is much smaller than the mean free path (which occurs with such high mobilities), it does mean that larger devices could be made which would have very low energy dissipation.

The energy dissipation for this type of reversible electronic logic (as estimated here) is greater than 
the dissipation of Drexler's rod logic[18] operated at a cycle time of 100 picoseconds (some 10 times faster than the 1 nanosecond considered here for the RCT). When RCT device operation using single electrons becomes feasible[4], then energy dissipation using RCT's would presumably be reduced. Likharev's parametric quantron has an energy dissipation which (when adjusted for the temperature difference and the differing speeds of operation) is not as good as rod logic. The parametric quantron also requires low temperature. Rod logic should work at room temperature. It is possible that mechanical molecular logic might have very favorable energy dissipation properties at low temperature[16].

While it is premature to draw conclusions it is not obvious that electronic devices must necessarily prove better than molecular mechanical devices in terms of energy dissipation at a given speed and temperature of operation.

\section{Clocking}

To insure that overall energy dissipation is reduced we need a method of providing suitable clocks that does not itself dissipate too much energy. We first consider a conceptually simple method of providing arbitrarily complex clock signals which can be made to dissipate as little energy as desired.

The loads presented to the clock in the proposals discussed here are purely capacitive. If we assume, for the moment, that the energy dissipated when a clock signal charges and discharges such purely capacitive loads is $1 / 2 \mathrm{CV}^{2}$, then the only method of reducing the energy dissipation is to reduce the voltage. Assume, for the moment, that we want a clock signal to increase from 0 volts to 5 volts. If we simply connect the clock line to a 5 volt source, then the energy dissipated will be $1 / 2 \mathrm{CV}^{2}=12.5 \mathrm{C}$ joules. If we have 10 voltage supplies, with voltages of $0.5,1.0,1.5,2.0,2.5$, $3.0,3.5,4.0,4.5$, and 5.0 volts, then we can successively connect our clock line to each voltage supply in turn. The voltage to the clock line would then be stepped up in 0.5 volt increments, and so the energy dissipated by a single step would be $1 / 2 \mathrm{C}(\mathrm{V} / 10)^{2}=0.125 \mathrm{C}$ (e.g., the energy dissipation per step is decreased by a factor of 100 because the size of the voltage step is decreased by a factor of 10).

To increase the voltage from 0 to 5 volts involves 10 steps, and so we will dissipate $10 \times(0.125 \mathrm{C})$ or $1.25 \mathrm{C}$ joules. This is 10 times smaller than the $12.5 \mathrm{C}$ joules we would have dissipated using a single 5 volt supply. Thus, be using 10 DC power supplies and switching the clock line successively from one power supply to the next, we have reduced energy dissipation by a factor of 10 . Clearly, we can reduce energy dissipation by a factor of $\mathrm{N}$ if we use $\mathrm{N}$ DC power supplies. Notice that the larger we make $\mathrm{N}$ the more time we must take to increase the voltage from 0 to 5 volts. Each time we take a step in voltage we must wait somewhat longer than one RC time constant for the voltage to settle at the new value. This settling time will remain about the same for each step, even as we decrease the size of the voltage step by increasing $\mathrm{N}$. As $\mathrm{N}$ increases and the number of steps increases, the total time taken for $\mathrm{N}$ steps will also increase.

This approach is very flexible because the clock waveform can rise and fall at arbitrary times. Complex clock signals can be easily generated.

There are many alternative clocking schemes. While the method just described is asymptotically 
nondissipative if a sufficiently large number of DC power supplies are used, it still involves moving electrons through wires to carry the clock signal throughout the circuit. This does not appear to be the major limiting factor in energy dissipation when using today's planar technology. However, if in the future we use single electron logic devices, then moving large numbers of electrons over long distances to provide the clock signals would seem inappropriate.

There are approaches to clock distribution which avoid the need to move charge through wires. A conceptually simple approach is to provide clock distribution by the use of charges that are fixed to a rotating tube or disk. A facing disk or a smaller concentric drum would carry the logic circuitry. As the disk spun the charges fixed to its surface would move, but this motion would not involve electrons flowing within a conductor. The electric charges on the surface of the disk would then move past the logic elements on the facing surface of the opposing disk, providing the repetitive voltage changes required to clock the logic circuitry. In principle this method of moving charges would result in no conventional resistive losses at all because the charge would not be moving through a conductor. There would still be radiative losses caused by the acceleration of the charged particles, and electrostatic attraction and repulsion would produce forces between the charges on the two disks which would lead to losses simply by alternately compressing and decompressing the material of the disk.

While this approach might not be attractive with current technology, it might prove attractive at some point in the future if strong materials could be rotated at high speeds, thus providing a high interfacial velocity between the two opposing surfaces; and the pattern of charges on the surface could be both precisely controlled and have high resolution. This should eventually be feasible[18, 32], though whether or not it will prove competitive with other approaches is uncertain.

Another method of providing a time-varying electric field would be to use a simple rotating electric field. Such a rotating electric field could then be used to clock the circuit. While the methods described here do not obviously lend themselves to such an approach, helical logic[67] uses such rotating electric fields to drive thermodynamically reversible logic operations. Charge is transported along a helix much as water is transported along an Archimedes screw. Single electron versions of helical logic should eventually be feasible. Rotating electric fields can be provided by several methods, including cavity resonators or multiple LC oscillators connected to plates arranged in an appropriate pattern in space.

\section{Other RCT's}

The 3-bucket RCT is not the only type of RCT that is useful. For example, if we use two condition buckets $\mathrm{C} 1$ and $\mathrm{C} 2$, and require that the charge packets in $\mathrm{C} 1$ and $\mathrm{C} 2$ be complementary (e.g., $\mathrm{C} 1$ is the negation of $\mathrm{C} 2$ ); and if we use a single source bucket $\mathrm{S}$ and two destination buckets D1 and D2; then we can implement the primitive "Transfer charge from S to D1 if C1 is false, but from S to D2 if $\mathrm{C} 1$ is true." We will again need a pre-condition that effectively prevents the merging of two packets; we omit the precise statement of this precondition. This 5-bucket primitive is logically complete (we leave this as an exercise for the reader) and has certain implementation advantages. It is illustrated in Figure 15. In particular, because both $\mathrm{C} 1$ and its complement $\mathrm{C} 2$ are available, we need only "steer" the charge in the source bucket to either of the destination buckets. We need not "bottle up" the charge on the source bucket in the event the transfer is not to take place (as occurs with the 3-bucket RCT). Because the charge packet in bucket $\mathrm{S}$ is guaranteed to move 


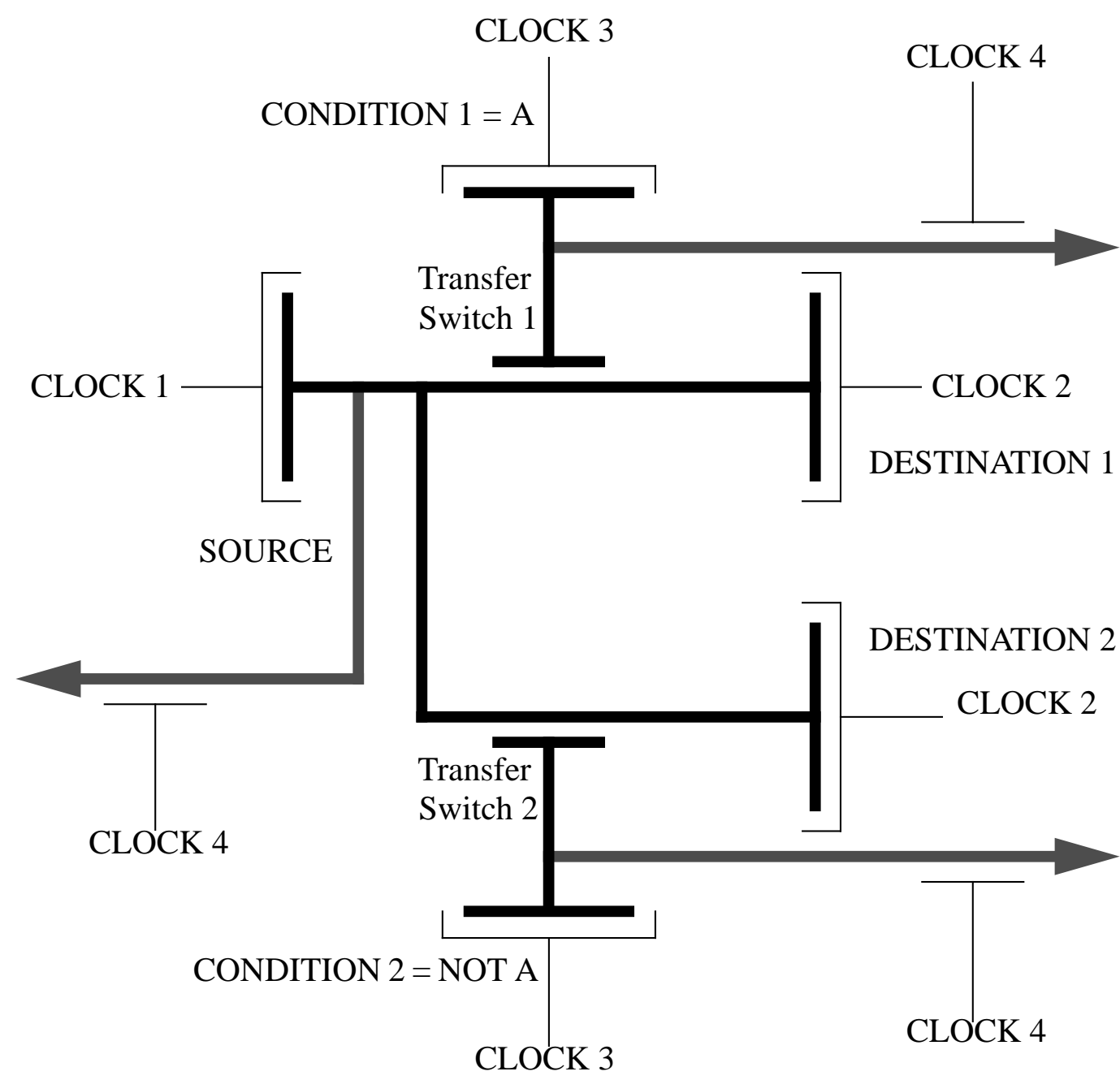

Figure 15

A 5-bucket RCT

Charge flows either to

Destination 1 or 2 .

Condition 1 and 2

are Complimentary

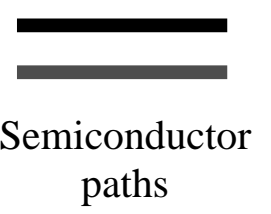

Conventional

wire

to some destination bucket (either D1 or D2), the actual voltage that must be generated to prevent the charge from moving in the wrong direction is smaller than the voltage required in the simple RCT to prevent the charge from transferring to its single destination bucket.

The same logic function could also be implemented in a somewhat different fashion by using a 4bucket RCT with a single source bucket $\mathrm{S}$, a single condition bucket $\mathrm{C}$ and two destination buckets D1 and D2. This is illustrated in Figure 16. In this alternative implementation, the charge in C 


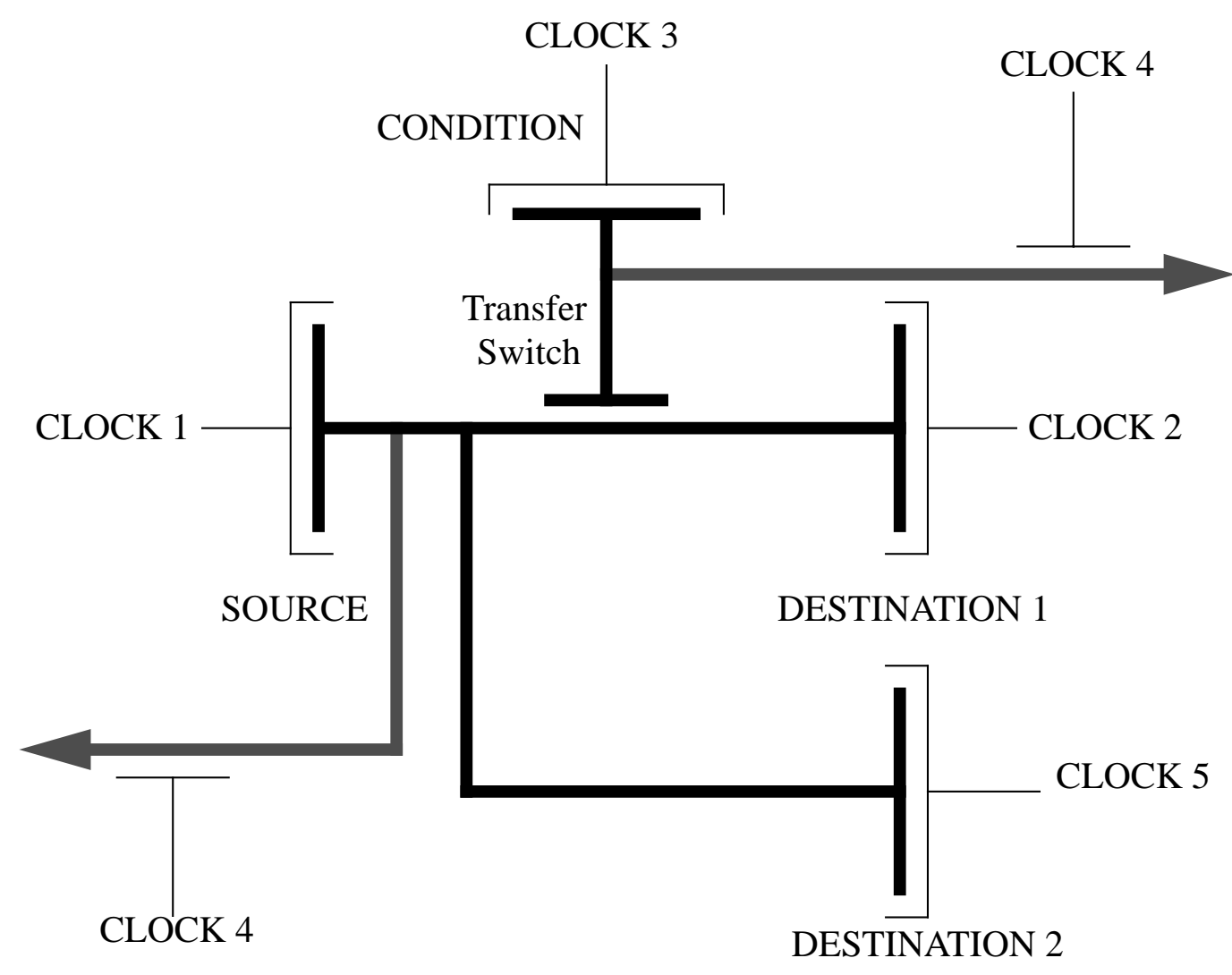

Figure 16

A 4-bucket RCT

After trying to transfer charge from

Source to Destination 1, any remaining

charge is transferred to Destination 2.

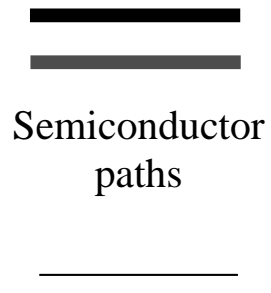

Conventional
wire

would control a single FET between S and D1, while S and D2 would always be connected. The key to the conditional transfer is the timing of the clock lines for the two destinations D1 and D2. In particular, we would transfer charge to D1 through the FET first. During this transfer, D2 would be maintained in an unreceptive state (the clock for D2 would be negative). After transfer of charge to D1 through the FET, the clock for D2 would be made positive. This shift would cause charge to transfer from S to D2 only if the charge had not previously been transferred to D1 through the FET. Thus, charge would be moved conditionally to either D1 or D2 depending on the condition C. In some sense, this is logically equivalent to the simple 3-bucket RCT operation followed by the transfer of the charge in S to the second destination bucket D2.

A 4-bucket RCT with a single source bucket $\mathrm{S}$, a single destination bucket $\mathrm{D}$ and two condition buckets $\mathrm{C} 1$ and $\mathrm{C} 2$ could be used to transfer charge only if both $\mathrm{C} 1$ and $\mathrm{C} 2$ were at logic 0 . Arbi- 
trary switching functions could be imposed between the source and destination buckets, and the number of condition buckets could be increased arbitrarily.

It should be clear that a myriad variations on this theme are possible.

\section{A Planar Version of RCT Logic}

It would be advantageous to have a planar layout for an RCT that used only sine waves for clocking. This would be easy to implement in current planar technology, and the clock signals could be generated by LC oscillators. These constraints can be met. In particular, we start by considering a 3-phase CCD as illustrated in figure 16. The three clocks, $\Phi_{0}, \Phi_{120}$ and $\Phi_{240}$, represent three sine

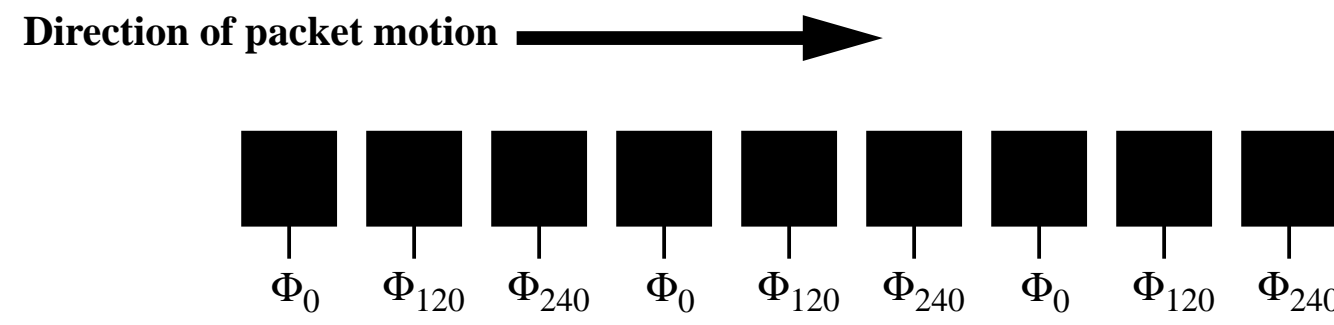

Figure 17

A 3-phase CCD

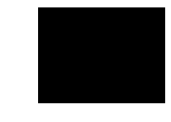

Metalization

waves offset by 0,120 , and 240 degrees respectively. Charge packets will move from left to right as the three clock signals vary sinusoidally over time. The illustration shows a top view, the black squares being the metalization regions. The metalization typically would be on top of $\mathrm{SiO}_{2}$, with the bulk Si beneath that. A profile view of charge transfer is given in figure 10.

To provide a switching operation, we must provide some sort of choice for the charge packets moving along the CCD. This is illustrated in figure 18, where the source CCD shift register divides into two offspring. To control the direction in which the charge packets are moved, we assume that two "condition" CCD shift registers provide the needed condition packets that will cause charge to select one offspring shift register over the other. Note that this device is just a 5-bucket RCT with a somewhat different geometry and clocking sequence. It uses 3-phase sine wave clocks to move charge through the device. An additional fourth phase $\left(\Phi_{180}\right)$ is required to control the conditional charge transfer from the single large source well (the large rectangle in the figure) to the two destination wells directly to its right.

In this particular planar RCT, Condition 1 and Condition2 are two CCD shift registers which hold complementary charges. If a potential well in the Condition1 shift register contains a charge packet, then the corresponding potential well in the Condition 2 shift register does not (and vice versa). The condition charge packet moves from left to right. When it reaches the well clocked by $\Phi_{180}$, the presence or absence of the charge packet changes the voltage on the corresponding gate electrode. There are two gate electrodes controlled by the two complementary charge packets. The 


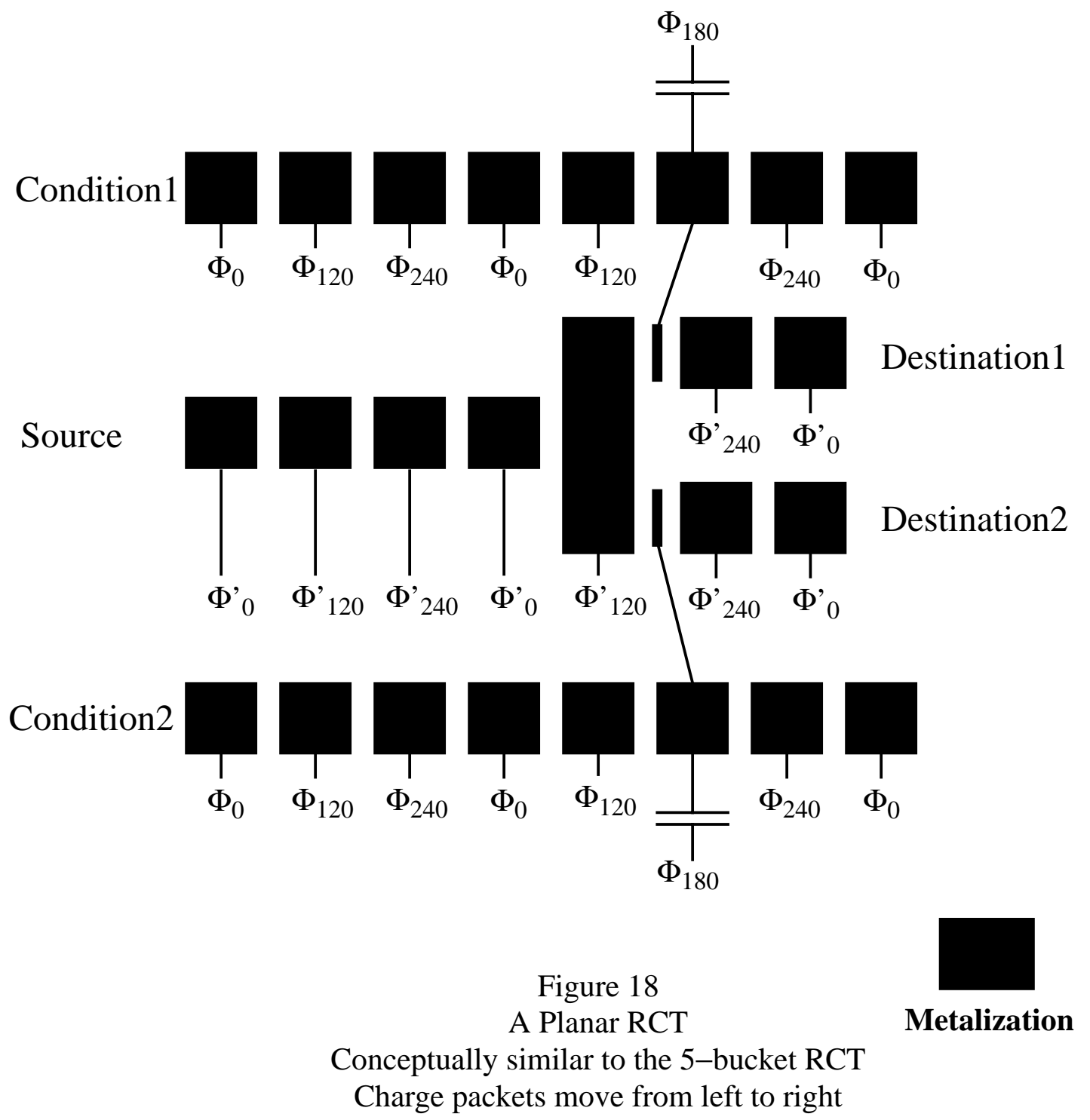

gate electrodes are between the source potential well (the large rectangular metalization) and the two destination potential wells to its right. The gate electrodes determine the destination well into which the charge packet in the source potential well moves. The voltage of the fourth phase, $\Phi_{180}$, is chosen so that the potential on the gate electrode is somewhat lower than the potential in the two adjacent potential wells when those two potentials are equal (e.g., when $\Phi_{120}=\Phi_{240}$ )and when the condition charge packet is absent. Thus, when the condition charge packet is absent, the charge packet in the source potential well will flow through the corresponding gate potential well and then into the corresponding destination potential well. When the condition charge packet is present, then the corresponding gate potential will be increased and the charge packet in the source potential well will be prevented from moving into the corresponding destination potential well. In this case the charge packet in the source potential well will find there is no barrier preventing it from moving into the other destination potential well.

Some clock signals are marked with a prime, as $\Phi$ '. The clock lines marked with a prime are the 
same as the those that are not so marked except for their DC bias level. Because the condition shift registers and the source/destination shift registers share the metalization for the $\Phi_{180}$ clock, and because basically different things must occur when a charge packet passes beneath the $\Phi_{180}$ metalization in the two cases, the DC bias levels for the two sets of clock signals need to be separately adjustable. Otherwise, when the gate electrode blocked a charge packet from moving from the source to the unselected destination, the same voltages applied to the metalizations on the corresponding condition shift register would likewise prevent the condition charge packet from moving along the condition shift register: this, of course, would cause problems. By adjusting the DC bias on the condition register clocks, we can insure that condition charge packets will move from left to right in a regular manner regardless of the voltage variation on the $\Phi_{180}$ clock caused by the presence or absence of the condition charge packet. In the same way, by adjusting the bias on the source/destination clocks, we can insure that the voltage variation on the gate electrodes will prevent charge packets from moving into the wrong destination, thus insuring reliable switching action.

In a real circuit, many RCT devices would be interconnected by a complex pattern of CCD shift registers. In most cases, this will force the data in one CCD shift register to cross over other shift registers. The use of a planar geometry makes the design of such a cross-over nonobvious. A relatively simple approach would be to use two crossing sets of metalizations which define two CCD shift registers, and then apply a bias voltage to inactivate one of the shift registers. A charge packet would be carried along the active shift register and would not be diverted into the inactive shift register because the bias voltage applied to the inactive shift register would eliminate the potential wells into which the charge packet might fall. By alternately inactivating one or the other shift register, charge packets could be carried across in first one and then the other direction. This can be likened to a traffic crossing with a traffic light, where two cars approaching at right angles cannot simultaneously occupy the intersection. The traffic light is first green in one direction, allowing one car to cross; and is then green in the other direction, allowing the other car to cross.

To summarize this planar RCT: a charge packet moving along a CCD shift register will be moved into one of two "offspring" or "descendant" shift registers. Which descendant is selected will depend on which of two condition shift registers is occupied by a charge packet. This operation is logically universal: it can be used to implement a Fredkin gate and therefore any reversible logic circuit. Just as this planar RCT is conceptually similar to a 5-bucket RCT, so too a planar version similar to the 4-bucket RCT would also be feasible and would have the practical advantage that only a single condition would be required to operate it. The planar 5-bucket RCT suggested here is intended to show that planar RCT's using simple sine waves for clocking are feasible. The design of an actual device might well differ significantly.

\section{Conclusions}

The historical trend in computer systems is to pack ever more logic gates into ever smaller volumes. This trend can only be continued if the energy dissipation per logic operation also continues to decline. The potential packing densities that nanoelectronic and molecular logic devices should be able to achieve will only be realized if the energy dissipated per logic operation can be reduced to extremely small values. Projections of current trends in energy dissipation per gate operation[6] suggest that the $k T$ "barrier" will become significant within ten to twenty years. This barrier can 
be overcome by using reversible logic. Reversible logic will be valuable well before the $k T$ barrier is reached. Even though not inherently required when the energy dissipation per logic operation is greater than $k T$, reversible designs can more easily reduce energy dissipation than irreversible designs even when the actual energy dissipation is orders of magnitude greater than $k T$. Two methods of electronic reversible logic using simple electronically controlled switches and capacitors were discussed. Both approaches can use FET-type switches in their operation. A planar implementation of the CCD based approach using simple sine waves for clocking should be particularly effective in achieving low energy dissipation per logic operation. Breaking the $k T$ barrier is feasible in principle, and will eventually be necessary if we are to continue the dramatic improvements in computer hardware performance and packing densities that we have seen during the last several decades. The ultimate limit for electronic devices will be reached when we are able to fabricate atomically precise logic elements that are thermodynamically reversible and use single electrons to represent information. This is likely to occur sometime early in the 21 st century.

\section{Acknowledgements}

The author would like to thank Bill Athas, Jim Burr, Tord Claeson, Eric Drexler, Edward Fredkin, Mike Hack, Jeff Koller, Rolf Landauer, Alan Lewis, Norman Margolus, Jim Mikkelsen, John Northrup, J. Storrs Hall, Bohumil Polata, Ed Richley, John Shaw, Jeff Soreff, Tommaso Toffoli, Bill Turner and Chris van de Walle.

\section{References}

1.) High-Speed Semiconductor Devices, by S. M. Sze, Wiley Interscience 1990.

2.) Rod Logic and Thermal Noise in the Mechanical Nanocomputer, by K. Eric Drexler, in Proceedings of the 3rd International Symposium on Molecular Electronic Devices, F.L. Carter, R.E. Siatkowski, H. Wohltjen, eds., North-Holland 1988.

3.) The Fundamental Physical Limits of Computation, by Charles H. Bennett and Rolf Landauer, Scientific American, July 1985, pages 48-56.

4.) Single Electronics, by Konstantin K. Likharev and Tord Claeson, Scientific American Vol 266 No. 6, June 1992, pages 80-85.

5.) The Fundamentals of Physics, Extended Third Edition, by David Halliday and Robert Resnick, Wiley 1988.

6.) Dissipation and noise immunity in computation and communication, by Rolf Landauer, Nature, Vol. 335, October 27 1988, page 779.

7.) Conservative Logic, by Edward Fredkin and Tommaso Toffoli, International Journal of Theoretical Physics, Vol. 21, Nos. 3/4, 1982, pages 219-253.

8.) The Thermodynamics of Computation - A Review, by Charles H. Bennett, International Journal of Theoretical Physics, Vol. 21, No. 12, 1982, pages 905-940. 
9.) Classical and Quantum Limitations on Energy Consumption in Computation, by K. K. Likharev, International Journal of Theoretical Physics, Vol. 21, Nos 3/4, 1982, pages 311 to 326.

10.) Reversible Conveyer Computation in Array of Parametric Quantrons, by K. K. Likharev, S. V. Rylov, and M. K. Semenov, IEEE Transactions on Magnetics, Vol. MAG-21, No. 2, March 1985, pages 947-950.

11.) Notes on the History of Reversible Computation, by Charles H. Bennett, IBM J. Research and Development, Vol. 32, No. 1, January 1988, pages 16-23.

12.) Engines of Creation, by K. Eric Drexler, Doubleday, 1986.

13.) There's Plenty of Room at the Bottom, a talk by Richard Feynman (awarded the Nobel Prize in Physics in 1965) at an annual meeting of the American Physical Society given on December 29, 1959. Reprinted in "Miniaturization", edited by H. D. Gilbert (Reinhold, New York, 1961) pages 282-296.

14.) A Small Revolution Gets Under Way, by Robert Pool, Science, Jan. 51990.

15.) Time/Space Trade-offs for Reversible Computation, by Charles H. Bennett, SIAM J. Computing, Vol. 18, No. 4, pages 766-776, August 1989.

16.) Two Types Of Mechanical Reversible Logic, by Ralph C. Merkle, submitted to Nanotechnology.

18.) Nanosystems: Molecular Machinery, Manufacturing, and Computation, by K. Eric Drexler, John Wiley, 1992.

19.) The Design of a Conservative Logic Computer and a Graphical Editor Simulator, by Andrew Lewis Ressler, Master's Thesis, Department of Electrical Engineering and Computer Science, Massachusetts Institute of Technology, January 1981.

20.) Donor Impurities, N-type Doping, and Impurity Diffusion in Diamond, by J. Bernholc, S. A. Kajihara, and A. Antonelli, 20th International Conference on Semiconductors, Volume 1, page 332-335.

21.) An Electroid Switching Model for Reversible Computer Architectures, by J. Storrs Hall, Laboratory for Computer Science Research, Rutgers University; presented at the Physics of Computation Workshop, Dallas Texas, Oct 2-4 1992.

22.) Quantum photoyield of diamond (111) -- - A Stable negative-affinity emitter, by F. J. Himpsel, J.A. Knapp, J.A. Van Vechten, and D. E. Eastman, Phys. Rev. B, Vol. 20 No. 2, pages 624-627.

23.) Design Principles for Achieving High-Performance Submicron Digital Technologies, by Edward Fredkin and Tommaso Toffoli, November 1978 DARPA proposal.

24.) Magnetic Bubbles, by Andrew H. Bobeck and H.E.D. Scovil, Scientific American, June 
1971, Vol. 224, No. 6, pages 78-91.

25.) Ideal MOS Curves for Silicon, by A. Goetzberger, Bell Systems Technical Journal, Vol 45, September 1966, pages 1097-1122

26.) Single atoms as transistors, by Sean Washburn, Nature Vol 357, May 21 1992, pages 199200.

27.) A CCD/CMOS process for integrated image acquistion and early vision signal processing, by Craig L. Keast and Charles G. Sodini, SPIE Vol. 1242 Charge-Coupled Devices and Solid State Optical Sensors (1990), pages 152-161.

28.) Eliminating Threshold Losses in MOS Circuits by Bootstrapping Using Varactor Coupling, by Reuben E Joynson, Joseph L. Mundy, and Constantine Neugebauer, IEEE Journal of SolidState Circuits, Vol SC-7, No. 3, June 1972, pages 217-224.

29.) Hot-Clock nMOS, by Charles L. Seitz, Alexander H. Frey, Sven Mattisson, Steve D. Rabin, Don A. Speck, Jan L.A. van de Sneepscheut, 1985 Chapel Hill Conference on VLSI, Computer Science Press, pages 1-17.

30.) Semiconductor Surface Varactor by R. Lindner, The Bell System Technical Journal, Vol. 41 No. 3, May 1962, pages 803-831.

31.) Energy-Time Trade-Offs in VLSI Computations, by Akhilesh Tyagi, pages 301-311; in Lecture Notes in Computer Science, 1989, Volume 405: Foundations of Software Technology and Theoretical Computer Science, Ninth Conference, Bangalore India, edited by C. E. Veni Madhaven, December 1989.

32.) A Proof about Molecular Bearings, by Ralph C. Merkle, submitted to Nanotechnology.

33.) Private communication, K. Eric Drexler.

34.) Digital Charge-Coupled Logic (DCCL), by T. A. Zimmerman, R. A. Allen, and Robert W. Jacobs, IEEE Journal of Solid State-Circuits, Vol. SC-12, No. 5, October 1977, pages 473-485.

35.) Combinatorial Digital Logic Using Charge-Coupled Devices, by J. Greg Nash, Journal of Solid-State Circuits, Vol SC-17, No. 5, October 1982, pages 957-963.

36.) Basic C.C.D. Logic Gates, by J. H. Montgomery and H. S. Gamble, The Radio and Electronic Engineer, Vol. 50, No. 5., pages 258-268, May 1980.

37.) A New Concept in Computing, by R. L Wigington, Proceedings of the IRE, April 1959, Vol 47, No 4, page 516-523.

44.) Approaching the Quantum Limit, by Karl Hess and Gerald J. Iafrate, IEEE Spectrum, Vol. 29 No. 7, July 1992, pages 44-49.

45.) Natural Josephson Junctions, by Colin Pegrum, Nature, Vol. 358, July 16 1992, page 193. 
46.) A Charge-Transfer-Device Logic Cell, by T. D. Mok and C. A. Salama, Solid State Electronics 1974, Vol. 17, pages 1147-1154.

47.) Charge Coupled Semiconductor Devices, by W. S. Boyle and G. E. Smith, Bell Sys. Tech. J., Vol. 49, pages 587-593, April 1970.

48.) A Simple Charge Regenerator for Use With Charge-Transfer Devices and the Design of Functional Logic Arrays, by Michael F. Tompsett, IEEE Journal of Solid-State Circuits, Vol. SC7, No. 3, June 1972, pages 237-242.

49.) Bucket-Brigade Electronics - New Possibilities for Delay, Time-Axis Conversion, and Scanning, by F.L.J. Sangster and K. Teer, IEEE Journal of Solid-State Circuits, Vol. SC-4, No. 3, June 1969, pages 131-136.

50.) Charge Transfer Devices, by M. F. Tompsett, J. Vac. Sci. Technol., Vol. 9, No. 4, July-August 1972, pages 1166-1181.

51.) Performance Tests of Large CCD's, by Lloyed Robinson, William Brown, Kirk Gilmore, Richard Stover, Mingzhi Wei, and John Geary,SPIE Vol. 1447 Charge Coupled Devices and Solid State Optical Sensors II (1991).

52.) The Role of Energy in VLSI Computation, by Akhilesh Tyagi, Ph.D. thesis, University of Washington, Computer Science, 1988.

53.) CMOS Differential Pass-Transistor Logic Design, by John H. Pasternak, Alex S. Shubat, and C. Andre T. Salama, IEEE Journal of Solid-State Circuits, Vol. SC-22, No. 2, April 1987, pages 216-222.

54.) Two-Dimensional Electron Gas Charge-Coupled Devices (2DEG-CCD's), by Eric R. Fossum, Jong-In Song, and David V. Rossi, IEEE Transactions on Electron Devices, Vol. 38, No. 5, May 1991, pages 1182-1192.

55.) A Unified Theory for MOS circuit design-switching network logic, by Min-You Wu, Wei Shu, and Shu-Park Chan, Int. J. Electronics, 1985, Vol. 58 No. 1, pages 1-33.

56.) Boolean Matrices and the Design of Combinational Relay Switching Circuits, by Franz E. Hohn and L. Robert Schissler, Bell System Technical Journal, January 1955, Volume 34, No. 1, pages 177-202.

57.) Introduction to VLSI Systems, by Carver Mead and Lynn Conway, Addison-Wesley 1980.

58.) Diamond Thin-Film Recessed Gate Field-Effect Transistors Fabricated by Electron Cyclotron Resonance Plasma Etching, by S. A. Grot, G. Sh. Gildenblat, and A. R. Badzian, IEEE Electron Device Letters, Vol. 13 No. 9, September 1992, pages 462-464.

59.) Low-Power CMOS Digital Design, by Anantha P. Chandrakasan, Samuel Sheng, and Robert W. Brodersen, IEEE Journal of Solid-State Circuits, Vol. 27, No. 4, April 1992, pages 473-484. 
60.) Course Notes on Program Inversion, by Jan van de Snepscheut, Caltech, 1992, to appear in Introduction to Computer Science, Spring Verlag, (David Gries's series) (in preparation).

61.) Adiabiatic Switching, Low Energy Computing, and the Physics of Storing and Erasing Information, by Jeffrey G. Koller and William C. Athas, Physics of Computation Workshop, October 2-4, Dallas Texas 1992.

62.) Packaging a $150 \mathrm{~W}$ Bipolar ECL Microprocessor, by William R. Hamburgen and John S. Fitch, WRL Research Report 92/1, March 1992, Digital Western Research Laboratory, Palo Alto, CA. Also published in 42nd Electronics Components and Technology Conference, Sand Diego, California, May 18-20 1992.

63.) Intel Takes Step To Get Computers To Save Energy, by Rose Gutfield, Wall Street Journal, Thursday October 15, 1992, page B6.

64.) High-performance Heat Sinking for VLSI, by D. B. Tuckerman and R. F. W. Pease, IEEE Electron Device Letters, Vol. EDL-2, No. 5, May 1981, pages 126-129.

65.) Upper and Lower Bounds on Switching Energy in VLSI, by Gloria Kissin, Journal of the ACM, Vol. 38, No. 1, January 1991, pages 222-254.

66.) A Symbolic Analysis of Relay and Switching Circuits, by Claude E. Shannon, Transactions of the American Institute of Electrical Engineers, Volume 57, 1938, pages 713-723.

67.) Helical Logic, by Ralph C. Merkle and K. Eric Drexler, in preparation.

68.) Edward Fredkin, private communication.

69.) Single Charge Tunneling, by H. Grabert and M.H. Devoret (eds), Plenum Press, 1992. 